\title{
Faint X\#Ray Sources in the Globular Cluster Terzan 5
}

\section{Citation}

Heinke, C. O., R. Wijnands, H. N. Cohn, P. M. Lugger, J. E. Grindlay, D. Pooley, and W. H. G. Lewin. 2006. "Faint X\#Ray Sources in the Globular Cluster Terzan 5." The Astrophysical Journal 651 (2): 1098-1111. https://doi.org/10.1086/507884.

\section{Permanent link}

http://nrs.harvard.edu/urn-3:HUL.InstRepos:41399938

\section{Terms of Use}

This article was downloaded from Harvard University's DASH repository, and is made available under the terms and conditions applicable to Other Posted Material, as set forth at http:// nrs.harvard.edu/urn-3:HUL.InstRepos:dash.current.terms-of-use\#LAA

\section{Share Your Story}

The Harvard community has made this article openly available.

Please share how this access benefits you. Submit a story.

Accessibility 


\title{
FAINT X-RAY SOURCES IN THE GLOBULAR CLUSTER TERZAN 5
}

\author{
C. O. Heinke, ${ }^{1,2}$ R. Wijnands, ${ }^{3}$ H. N. Cohn, ${ }^{4}$ P. M. Lugger, ${ }^{4}$ J. E. Grindlay, ${ }^{5}$ D. Pooley, ${ }^{6,7}$ and W. H. G. Lewin ${ }^{8}$ \\ Received 2006 February 22; accepted 2006 July 21
}

\begin{abstract}
We report our analysis of a Chandra X-ray observation of the rich globular cluster Terzan 5 , in which we detect 50 sources to a limiting 1.0-6 keV X-ray luminosity of $3 \times 10^{31} \mathrm{ergs} \mathrm{s}^{-1}$ within the half-mass radius of the cluster. Thirty-three of these have $L_{\mathrm{X}}>10^{32} \mathrm{ergs} \mathrm{s}^{-1}$, the largest number yet seen in any globular cluster. In addition to the quiescent low-mass X-ray binary (LMXB; identified by Wijnands et al.), another 12 relatively soft sources may be quiescent LMXBs. We compare the X-ray colors of the harder sources in Terzan 5 to the Galactic center sources studied by Muno and collaborators and find the Galactic center sources to have harder X-ray colors, indicating a possible difference in the populations. We cannot clearly identify a metallicity dependence in the production of lowluminosity X-ray binaries in Galactic globular clusters, but a metallicity dependence of the form suggested by Jordán et al. for extragalactic LMXBs is consistent with our data.
\end{abstract}

Subject headings: globular clusters: individual (Terzan 5) — novae, cataclysmic variables — stars: neutron $\mathrm{X}$-rays: binaries

Online material: color figures

\section{INTRODUCTION}

Globular clusters are highly efficient at producing X-ray binaries through dynamical interactions (Ivanova et al. 2005). For luminous low-mass X-ray binaries (LMXBs; for the purposes of this paper, all discussion of LMXBs refers to those containing accreting neutron stars), this has been known for many years, as their production rate per unit mass is more than 100 times that of the rest of the Galaxy (Clark 1975). Only in the past few years has it been possible to study the populations of faint X-ray sources in the densest globular clusters in depth, due to the high spatial resolution of the Chandra X-Ray Observatory and optical identifications by the Hubble Space Telescope (see Verbunt \& Lewin 2006 for a review). These low-luminosity X-ray sources include quiescent LMXBs (qLMXBs), identified by their previous outbursts or soft blackbody-like X-ray spectra (in 't Zand et al. 2001; Rutledge et al. 2002); cataclysmic variables (CVs), generally identified by their blue, variable optical counterparts (Cool et al. 1995); chromospherically active main-sequence binaries (ABs), identified by their main (or binary) sequence; variable optical counterparts (Edmonds et al. 2003); and millisecond pulsars (MSPs), identified by their spatial coincidence with radio timing positions (Grindlay et al. 2001a).

These lower luminosity X-ray sources are also produced through dynamical interactions, as demonstrated by the correlation between the number of X-ray sources in a cluster and its "collision

\footnotetext{
1 Department of Physics and Astronomy, Northwestern University, 2145 Sheridan Road, Evanston, IL 60208; cheinke@northwestern.edu.

2 Lindheimer Postdoctoral Fellow.

3 Astronomical Institute "Anton Pannekoek," University of Amsterdam, Kruislaand 403, 1098 SJ Amsterdam, Netherlands.

4 Department of Astronomy, Indiana University, Swain West 319, Bloomington, IN 47405.

5 Harvard-Smithsonian Center for Astrophysics, 60 Garden Street, Cambridge, MA 02138 .

6 Department of Astronomy, University of California, Berkeley, CA 947203411 .

7 Chandra Fellow.

8 Kavli Institute for Astrophysics and Space Research, Massachusetts Institute of Technology, Cambridge, MA 02139.
}

number" (Pooley et al. 2003), a measure of the cluster's stellar interaction rate. One of the clusters with the highest collision number is Terzan 5 , a dense cluster located $8.7 \mathrm{kpc}$ away, near the Galactic center (Cohn et al. 2002; Heinke et al. 2003a). This cluster hosts a transiently luminous LMXB, EXO 1745-248, first detected through X-ray bursts in 1980 (Makishima et al. 1981) and remaining irregularly active since then (Wijnands et al. 2005). Terzan 5 also hosts at least 30 MSPs, the largest number yet discovered in any globular cluster, and the fastest known MSP (Ransom et al. 2005; Hessels et al. 2006). Terzan 5 also has a high metallicity of $[\mathrm{Fe} / \mathrm{H}]=-0.21$ (Origlia \& Rich 2004).

The incidence of bright LMXBs in globular clusters has been clearly associated with increasing metallicity, but to date the effects of metallicity of faint X-ray binaries in globular clusters have not been studied. Grindlay (1987) identified an apparent trend for LMXBs to be more common in metal-rich globular clusters in the Milky Way, confirmed for the Milky Way and M31 by Bellazzini et al. (1995). Kundu et al. (2002) demonstrated that metal-rich clusters are 3 times more likely than metal-poor clusters to possess LMXBs in the elliptical NGC 4472. This result has been confirmed for various early-type galaxies by Maccarone et al. (2003), Kundu et al. (2003), Sarazin et al. (2003), and Jordan et al. (2004), the last offering a scaling for the likelihood of a cluster in M87 hosting an LMXB of $\left(Z / Z_{\odot}\right)^{0.33 \pm 0.1}$. Suggested explanation for this effect are a dependence of the cluster initial mass function on the metallicity (Grindlay 1987), a change in the rate of tidal captures (Bellazzini et al. 1995), a change in the strength of stellar winds (Maccarone et al. 2004), and a change in magnetic breaking rates due to differences in convective zone depths (Ivanova 2006).

Terzan 5 was observed by Chandra in 2000 (two closely spaced observations) and 2002. The 2000 observations caught EXO 1745-248 during a bright outburst (Heinke et al. 2003a). The high resolution of Chandra allowed the detection of nine additional low-luminosity sources within the cluster, and some useful spectral information on the transient was recovered from the readout streak. In the 2002 observation, EXO 1745-248 was observed at a typical X-ray luminosity for a $\mathrm{qLMXB}\left(L_{\mathrm{X}}=2 \times\right.$ $10^{33} \mathrm{ergs} \mathrm{s}^{-1}$ ) but with an unusually hard X-ray spectrum (Wijnands 
TABLE 1

X-Ray SOURCes in TERZAN 5

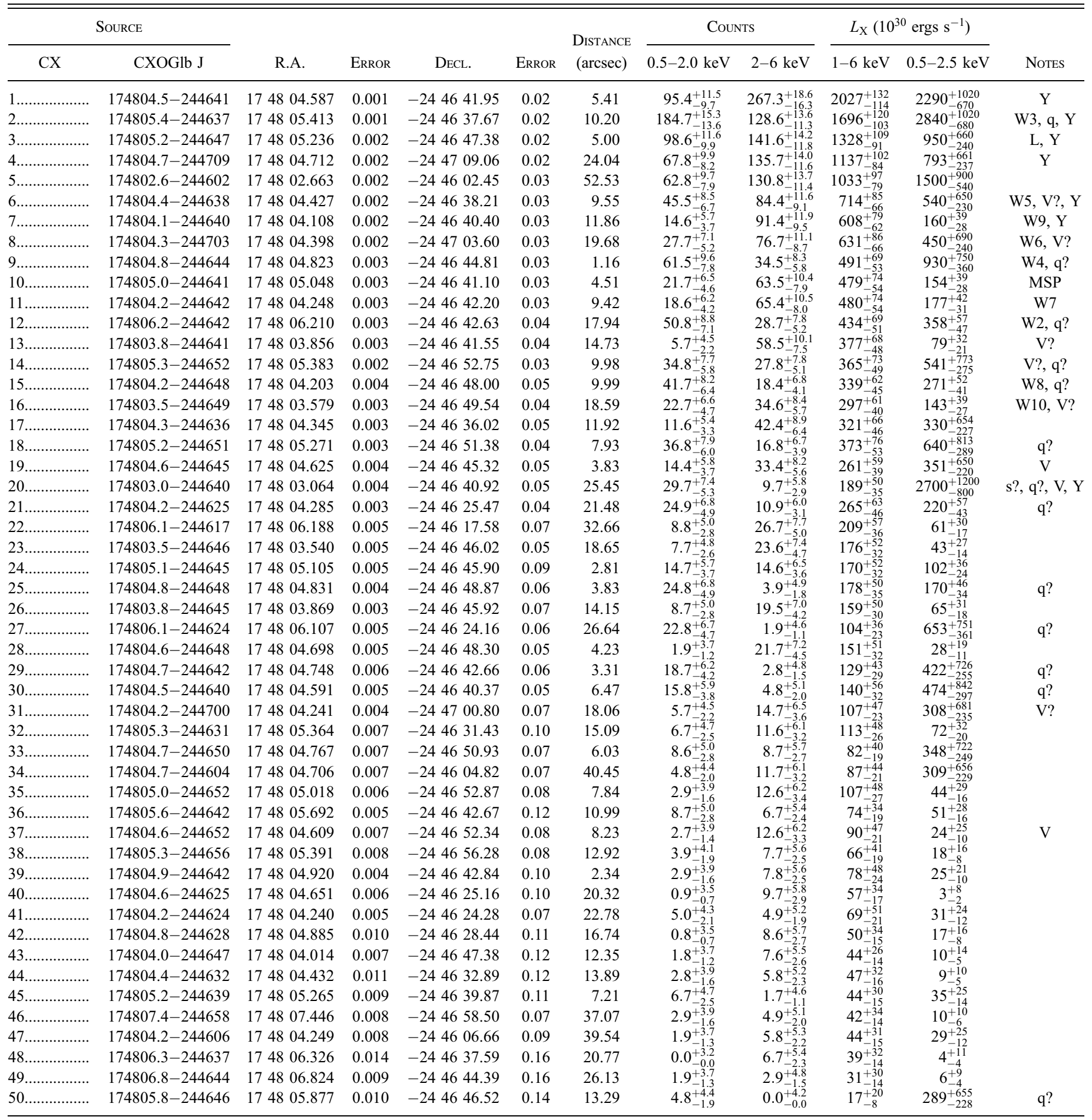

Notes.-Units of right ascension are hours, minutes, and seconds, and units of declination are degrees, arcminutes, and arcseconds. Given are the names, positions, distance from the center of Terzan 5, counts in two X-ray energy bands, and estimated X-ray luminosities of X-ray sources associated with Terzan 5. The errors in the position represent the $1 \sigma$ uncertainties in the relative positions of the sources, derived from ACIS_EXTRACT centroiding. The counts in each band are the numbers of photons within the source regions of Fig. 1. Luminosities are computed from the corrected photon fluxes in several narrow bands (see text). Notes indicate short-term variability ( $\mathrm{V}=99 \%$ confidence; $\mathrm{V}$ ? = 95\% confidence), variability on timescales of years between 2000 and 2003 ( $\mathrm{Y}$ ), and possible identifications ( $\mathrm{L}$ : transient $\mathrm{LMXB}$ EXO 1745-248; q: qLMXB; q: qLMXB candidate; s: foreground star; MSP: radio millisecond pulsar). 


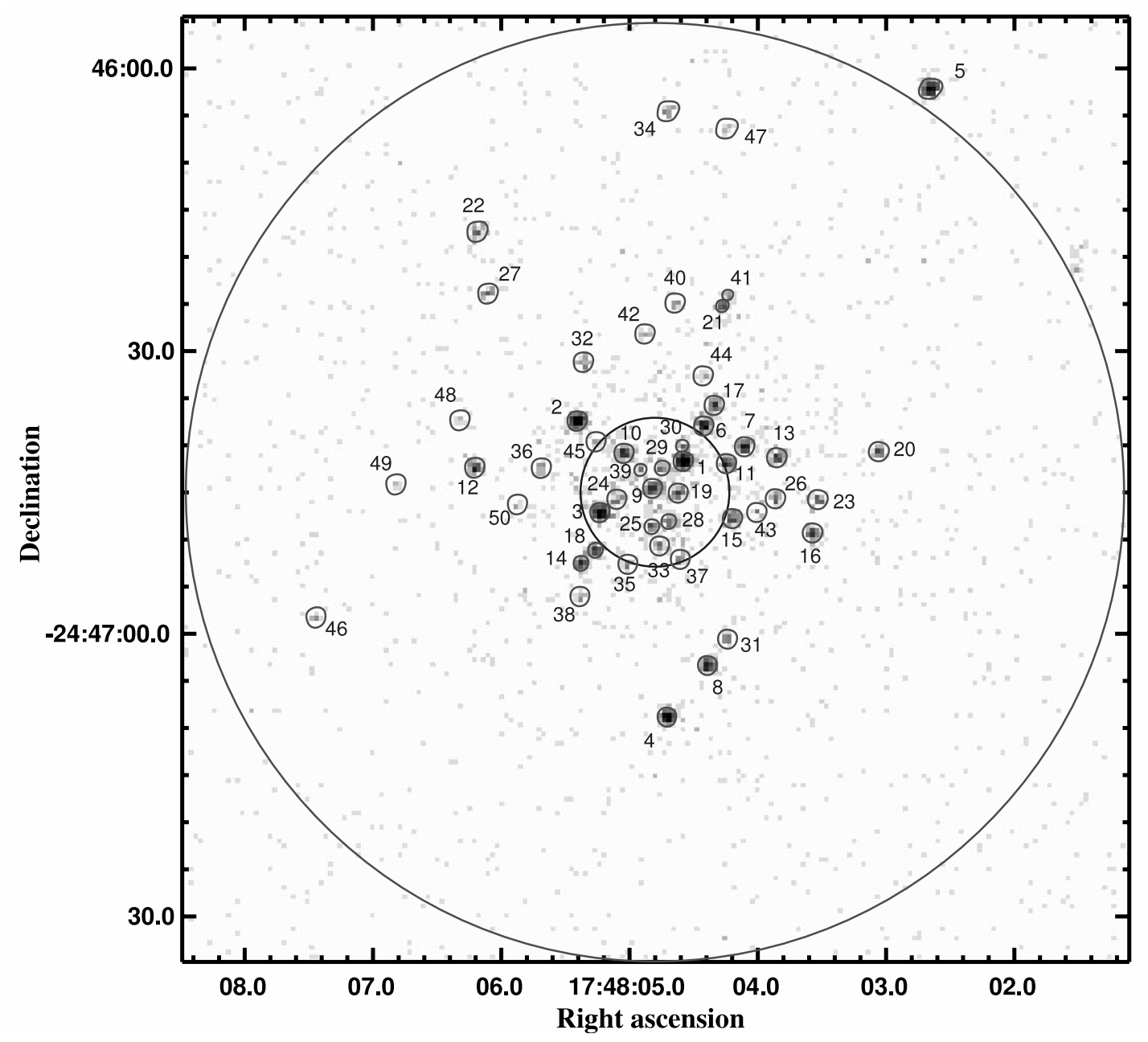

Fig. 1.-X-ray image of Terzan 5, with the core (7."9) and half-mass radii marked and the extraction regions for the sources associated with Terzan 5. [See the electronic edition of the Journal for a color version of this figure.]

et al. 2005). Wijnands et al. (2005) also detected a large number of faint X-ray sources in Terzan 5, which are the focus of this paper.

\section{OBSERVATIONS AND REDUCTION}

We observed Terzan 5 with Chandra for $39.3 \mathrm{ks}$ on 2003 July 13-14 using the Advanced CCD Imaging Spectrometer (ACIS) S3 chip (turning off other chips to avoid the possibility of telemetry saturation in case of an LMXB outburst). We reduced and analyzed the data using the Chandra Interactive Analysis of Observations (CIAO), version 3.2.1 software. $^{9}$ We reprocessed the level 1 event files using the latest (time dependent) gain files, using bad pixel files generated with the new acis_run_hotpix routine, and without the pixel randomization that is applied in standard data processing. We filtered on grade, status, and good time intervals supplied by standard processing. The later part of the observation suffers from elevated background levels. We removed $4.0 \mathrm{ks}$ of data with background flares, for a total good time of $35.3 \mathrm{ks}$.

\subsection{Detection and Source Property Extraction}

We focus our analysis on the sources (with one exception that we discuss below) within the cluster half-mass radius $\left(r_{h}=\right.$ 0.83; Harris 1996, updated in 2003), as done for other clusters (e.g., Pooley et al. 2003; Heinke et al. 2005b). This offers an excellent balance between including most cluster sources and excluding most background sources. Since globular cluster X-ray sources are generally more massive than the typical cluster star, they tend to concentrate toward the core of dynamically relaxed clusters such as Terzan 5 .

We selected an energy band of $0.5-7.0 \mathrm{keV}$ to search for sources with maximum sensitivity while minimizing the background. We ran two wavelet detection algorithms, the CIAO task wavdetect (Freeman et al. 2002) and the pwdetect ${ }^{10}$ algorithm (Damiani et al. 1997), on ACIS chip S3, with broadly similar results. We find that pwdetect is somewhat more effective at identifying faint sources very near brighter sources, while wavdetect is more reliable over large fields; we thus adopt pwdetect results within $r_{h}$ and wavdetect results otherwise. We choose the sensitivity of our detection algorithms to identify no more than one spurious source within $r_{h}$ (for pwdetect) and the $\mathrm{S} 3$ chip (for wavdetect). We find a total of 49 sources at or within the cluster $r_{h}$. One bright source (CXOGlb J174802.6-244602) located just beyond $r_{h}$ seems highly likely to be associated with the cluster (due to its high flux; its X-ray colors are consistent with either an active galactic nucleus [AGN] or CV), and we include it in our analyses of the cluster sources as well. We tabulate properties of the likely cluster sources in Table 1 and of other sources 
TABLE 2

Serendipitous Sources in the Terzan 5 Field

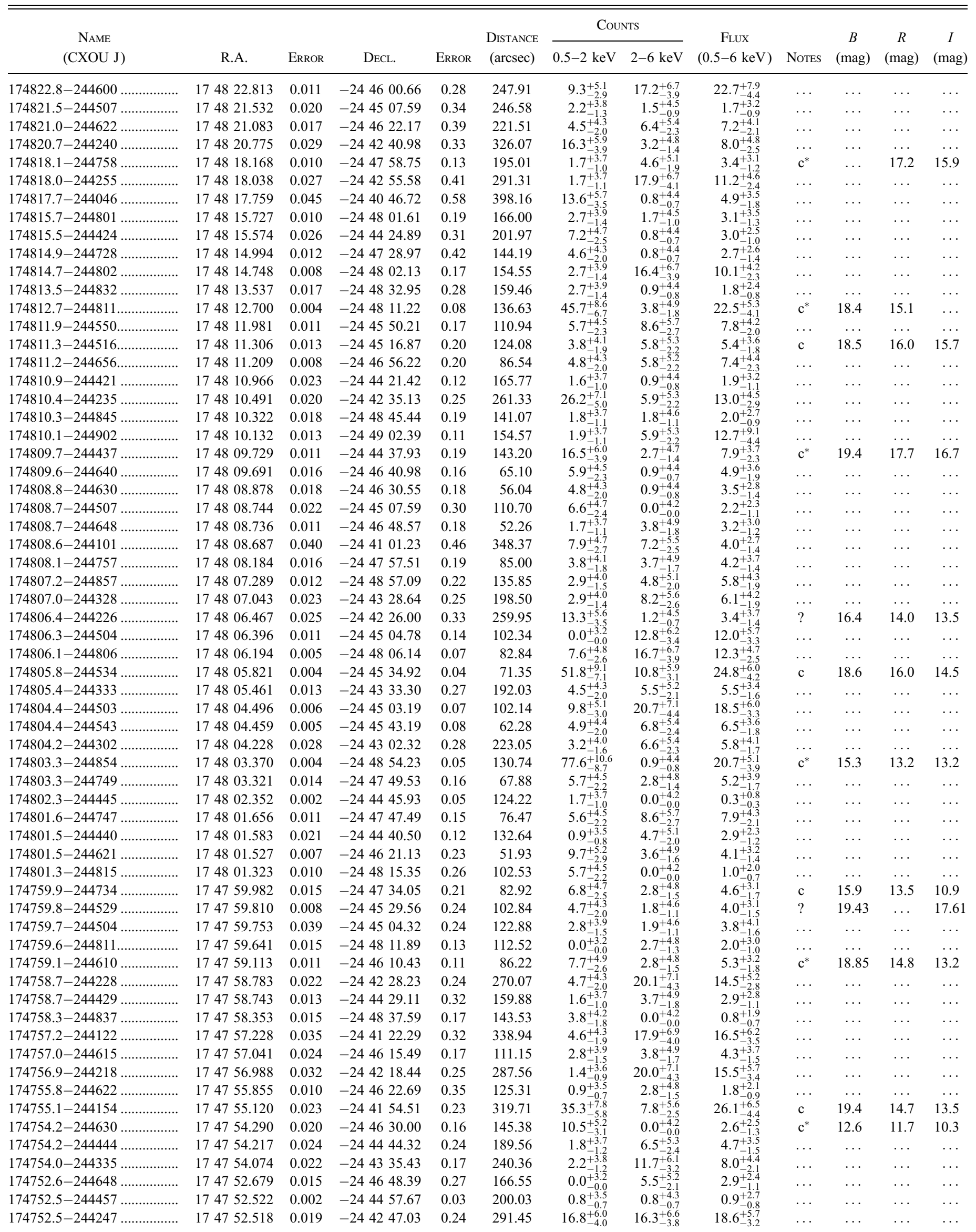


TABLE 2-Continued

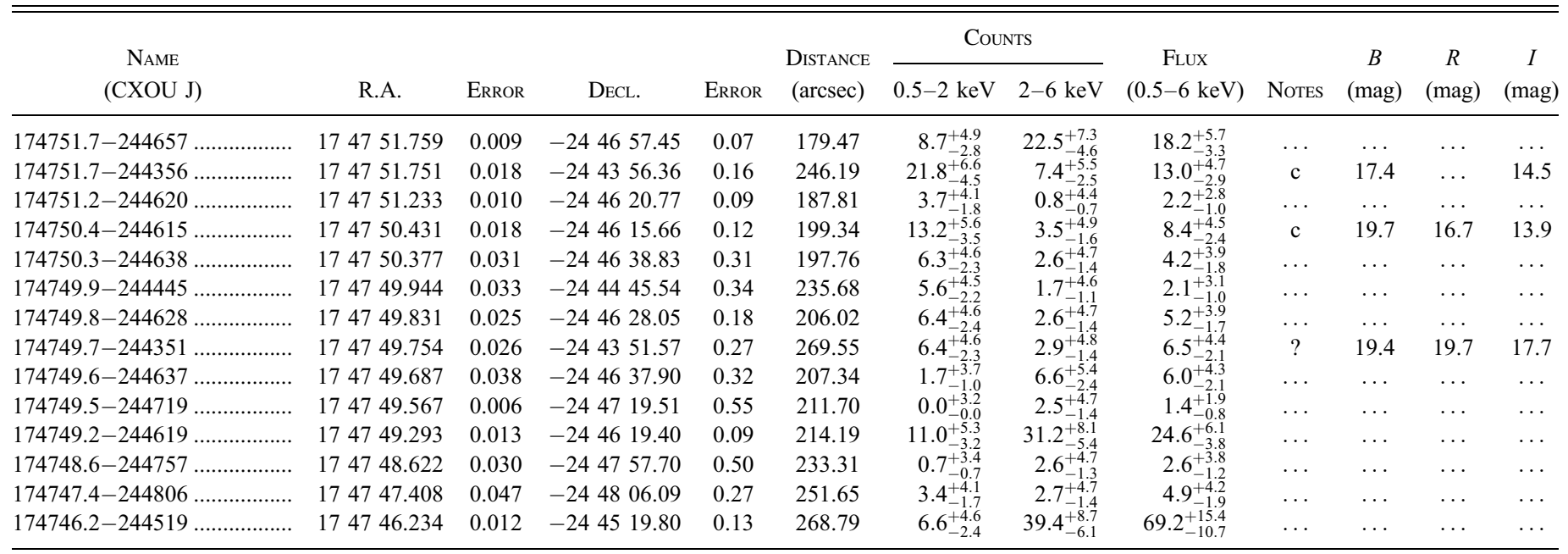

Notes.-Sources outside the Terzan 5 half-mass radius detected on the S3 chip. Units of right ascension are hours, minutes, and seconds, and units of declination are

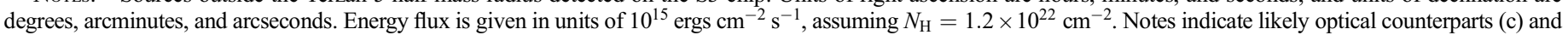

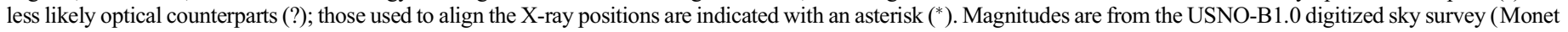
et al. 2003), averaged when two measurements exist; note that there is substantial scatter among these measurements (up to 1 mag), presumably due to crowding.

in the field in Table 2. In Figure 1, we show a $0.5-7 \mathrm{keV}$ Chandra image of Terzan 5, including the extraction regions for our identified sources.

To extract source photometry and spectra and improve source positions, we use the IDL program ACIS_EXTRACT ${ }^{11}$ (Feigelson et al. 2002). This program automates the extraction process using CIAO and FTOOLS software and is designed for working with crowded fields and multiple observations. The principal benefits of this software were its production of extraction regions designed to match the contours for a point-spread function (PSF) fraction of the user's choice and application of this PSF fraction to results from photometry and spectroscopy. We briefly describe the major elements of this process.

We refined the positions (starting from the pwdetect positions) of all sources within the cluster by finding the centroid of the detected counts within the ACIS_EXTRACT-produced extraction radii. We extracted counts from within the $90 \%$ contour for most sources, choosing the $95 \%$ contour for relatively bright and isolated sources and smaller contours for faint sources experiencing heavy crowding. We note that the sources within the cluster core radius are likely to suffer some degree of confusion. We compute background-subtracted photometry for each source, accounting for the fraction of the PSF enclosed and for the energy dependence of this fraction. To compute fluxes (for the $0.5-2.5$ and $1.0-6 \mathrm{keV}$ bands), we find the count rates observed in several narrow bands $(0.5-1,1.0-1.5,1.5-2.0,2.0-2.5,2.5-3.3,3.3-$ $4.5,4.5-4.7$, and $4.7-6.0 \mathrm{keV})$ and compute conversion factors from photon fluxes to unabsorbed energy fluxes for each band (assuming $N_{\mathrm{H}}=1.2 \times 10^{22} \mathrm{~cm}^{-2}$; Cohn et al. 2002), then sum the energy fluxes. We extract background spectra using regions sized to include $>100$ counts and excluding mask regions around identified sources. Response matrices are constructed using the CIAO tool mkacisrmf, and effective area files are modified to account for the energy-dependent aperture corrections (by ACIS_EXTRACT).

The substantial column density in the direction of Terzan 5 $\left(1.2 \times 10^{22} \mathrm{~cm}^{-2}\right)$ is likely to obscure background AGNs and reduce their number counts. We expect approximately eight back-

\footnotetext{
${ }^{11}$ Available at http://www.astro.psu.edu/xray/docs/TARA/ae_users_guide
}

ground AGNs with more than 10 counts on the S3 chip outside $2 r_{h}$ from Terzan 5 (based on the results of Giacconi et al. [2001] and including the effects of absorption), but we see 22 such sources. This indicates a population of Galactic sources, including foreground stars and possible faint CVs in the Galactic bulge (Grindlay et al. 2002). Therefore, we estimate our noncluster source numbers by looking at the radial distribution of X-ray sources. Outside a radius of $2 r_{h}$ from Terzan 5, the numbers of X-ray sources above 10 counts are at 0.4 arcminute $^{-2}$, implying that $\sim 1$ of 37 such sources within $r_{h}$ is not associated with the cluster. For the $1 r_{h}-2 r_{h}$ annulus, 3.4 of 6 sources above 10 counts are likely to be associated with the cluster, and for the $2 r_{h}-3 r_{h}$ annulus only 1.6 of 6 sources may be cluster members. Foreground chromospherically active stars

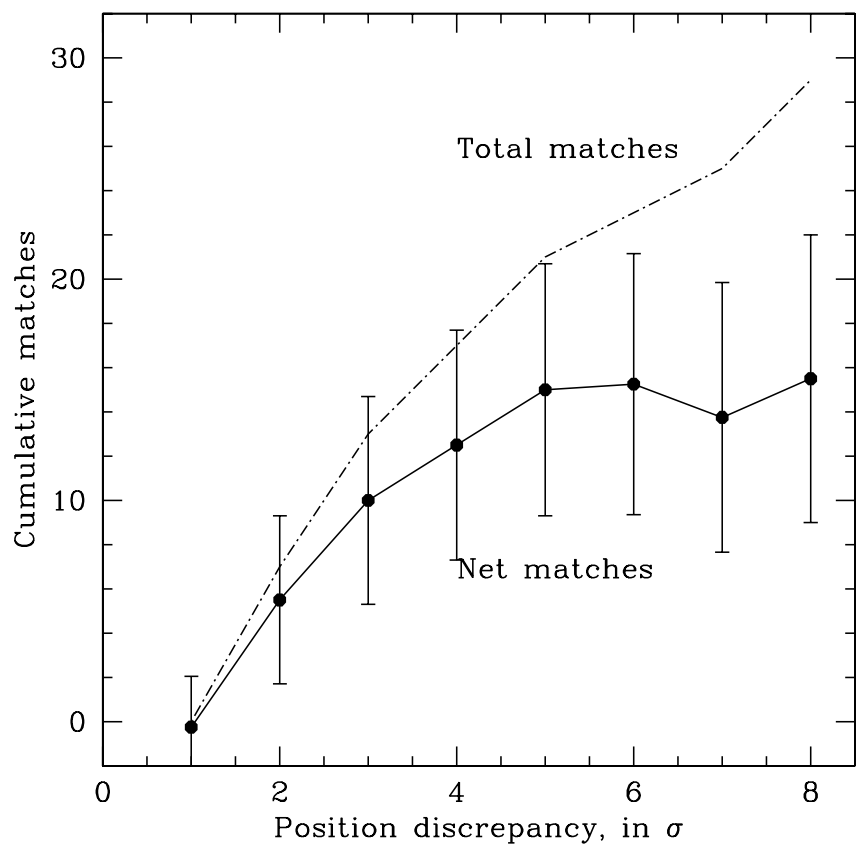

FIG. 2.- Numbers of possible optical counterparts outside the Terzan 5 halfmass radius (dash-dotted line, total; solid line, net, after subtraction of average number of matches with $15^{\prime \prime}$ shifts) vs. search radius (in units of $\sigma$, combined Chandra and USNO errors). 

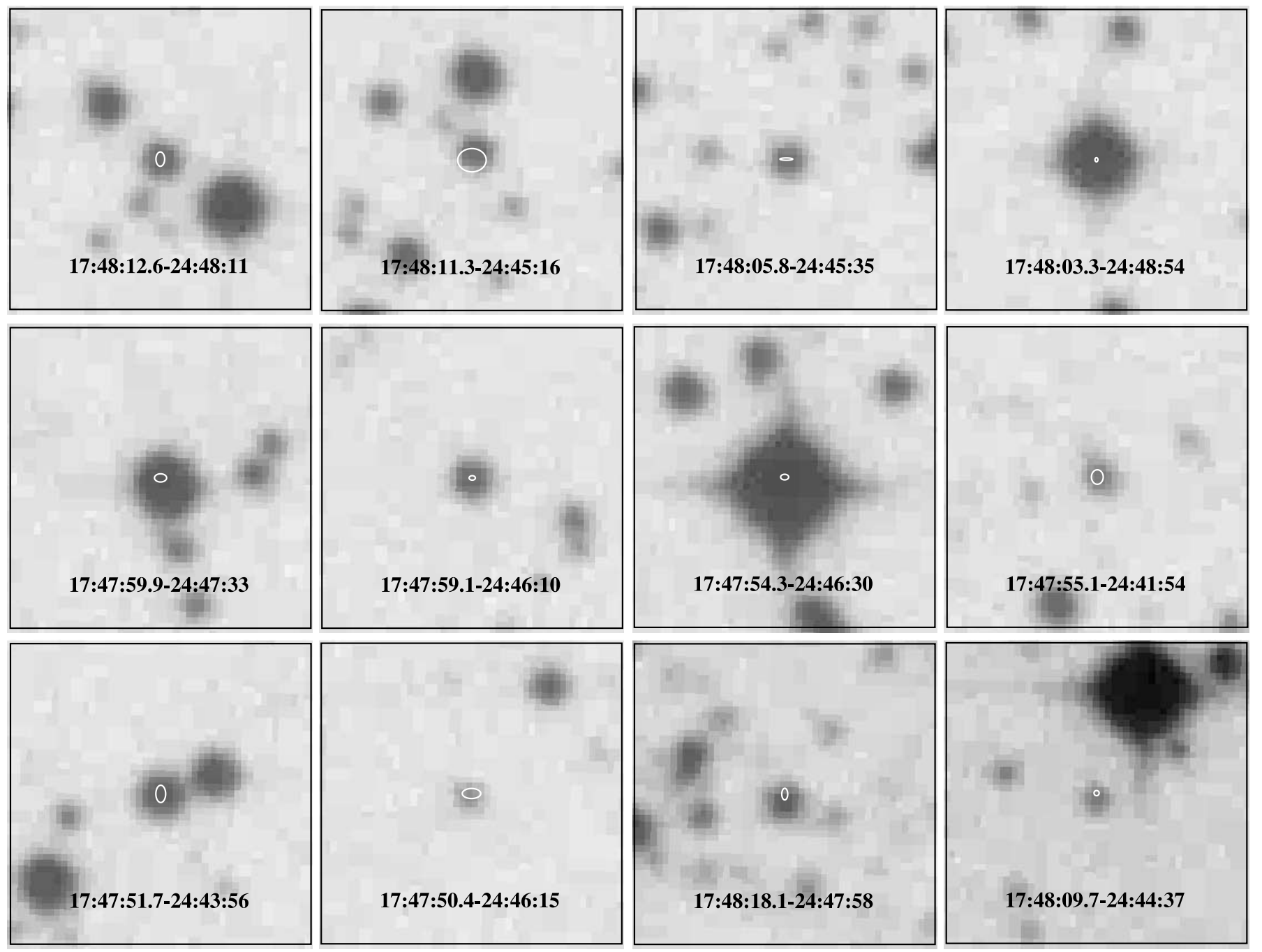

FIg. 3.- Likely optical counterparts outside the Terzan 5 half-mass radius. Ellipses represent twice the summed USNO and Chandra uncertainties. Each box is $33^{\prime \prime}$ on each side. Optical images are from the UKST $B$ image, except CXOU J174818.1-244758, for which the UKST $I$ image is used.

may be identifiable through optical counterparts or very soft spectra, while background AGNs and distant CVs may be indistinguishable.

\section{ANALYSIS}

\subsection{Astrometry and Possible Counterparts}

We searched for possible optical counterparts to sources outside $r_{h}$ using the USNO-B1.0 catalog (Monet et al. 2003). We identify several probable optical counterparts, of which we take six relatively secure and uncrowded matches (marked with an asterisk in Table 2) to define our reference frame. To match the USNO-B1.0 frame, we shift our Chandra positions by +0 ".083 $( \pm 0.06)$ in right ascension and $-0.175( \pm 0.06)$ in declination. Defining $\sigma$ as the sum in quadrature of the USNO uncertainties (typically $0.06-0.3)$ and the centroiding errors derived from ACIS_EXTRACT, we measured the numbers of possible counterparts within 1-8 $\sigma$ and compared these to the numbers of spurious counterparts found by shifting the Chandra positions $5^{\prime \prime}$ in four directions (Fig. 2). (We exclude faint USNO stars with large quoted errors of $0^{\prime \prime} .999$ and matches within three core radii of Terzan 5 due to optical crowding.) We find that real matches occur for separations up to $\sim 4 \sigma$, within which $12.5 \pm 5.2$ of the 17 total matches represent an excess over the expected number of spurious matches. Systematic errors (due to optical crowding), un- measured proper motions, and binaries with fainter stellar companions may contribute to generating these apparently large errors.

We carefully inspected United Kingdom Schmidt Telescope (UKST) $B$ and $I$ plates of the region (Hambly et al. 2001). ${ }^{12} \mathrm{We}$ identify 12 very likely optical counterparts, for which we show postage stamps in Figure 3, and three other possible matches.

These 15 matches are marked as " $c$ " in the notes to Table 2, including two counterparts discussed in Heinke et al. (2003a). Three other possible matches are marked with a question mark. We note that the sources with optical counterparts include the six brightest sources (beyond Terzan 5's $r_{h}$ ) below $2 \mathrm{keV}$ and that their spectra are generally much softer than those of other sources, as expected for typical stars.

\subsection{X-Ray Color-Magnitude Diagram}

X-ray color-magnitude diagrams (XCMDs), plotting an X-ray color against detected counts or X-ray luminosity, have been used by several authors as an effective way to understand the source populations in globular clusters (Grindlay et al. 2001a; Pooley et al. 2002a; Heinke et al. 2003c). Due to the high

\footnotetext{
${ }^{12}$ We used SuperCOSMOS via Aladin; see http://aladin.u-strasbg.fr/aladin gml.
} 


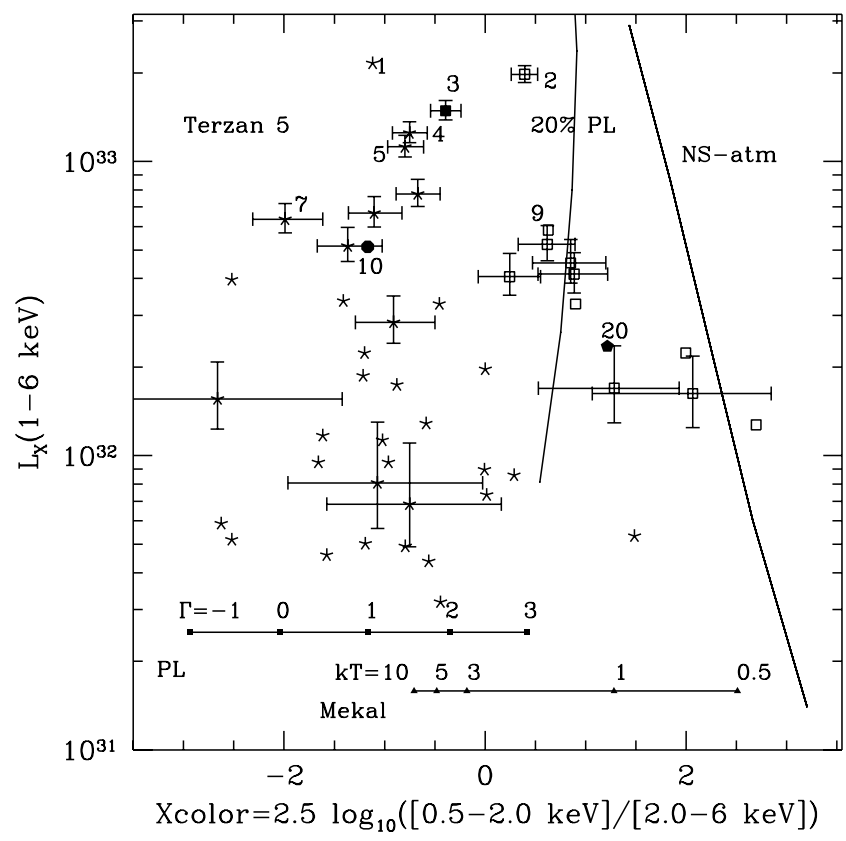

FIG. 4.-X-ray CMD of Terzan 5, plotting X-ray luminosity $(1.0-6 \mathrm{keV}$, corrected for extinction of $\left.1.2 \times 10^{22} \mathrm{~cm}^{-2}\right)$ against an X-ray color $(2.5 \log [(0.5-$ $2.0 \mathrm{keV}) /(2.0-6.0 \mathrm{keV})]$, thus hardening to the left) for sources within the halfmass radius of Terzan 5 (plus CXOGlb J174802.6-244602; see text). X-ray luminosities are computed assuming a $6 \mathrm{keV}$ MEKAL spectrum to convert photon fluxes into energy fluxes for each of eight narrow bands and summed. Several sources of particular interest (including the X-ray sources identified by Heinke et al. 2003a) are identified with their number. The filled square represents a probable qLMXB, the open squares possible qLMXBs, the filled circle a likely millisecond pulsar, the pentagon a possible foreground star or qLMXB (see $\S 3.5 .3$ ), and the asterisks sources of unknown nature. See the text for a description of the model tracks. [See the electronic edition of the Journal for a color version of this figure.]

absorption toward Terzan 5, several X-ray sources have no detected counts below $1.5 \mathrm{keV}$. To avoid displaying large numbers of upper limits, we therefore choose a nonstandard X-ray color, $2.5 \log [(0.5-2.0 \mathrm{keV}) /(2.0-6.0 \mathrm{keV})]$. The extrapolation from a few detected $0.5-1 \mathrm{keV}$ counts to the $0.5-1 \mathrm{keV}$ luminosity is extremely uncertain, so we choose to compute and plot more accurate 1-6 keV luminosities. This reduces the size of the luminosity errors by up to $90 \%$ in some cases. We plot the XCMD in Figure 4 versus the 1.0-6.0 keV X-ray luminosity inferred (assuming $N_{\mathrm{H}}=1.2 \times 10^{22} \mathrm{~cm}^{-2}$ ) for each source (see above).

To make our XCMD useful for comparison with other clusters, we plot on this XCMD the X-ray colors of power-law (PL) and thermal plasma (MEKAL in XSPEC; Liedahl et al. 1995) spectral models. We also plot the expected location of neutron stars with hydrogen atmospheres radiating away stored heat (labeled "NS-atm"; Heinke et al. 2006), as expected for quiescent LMXBs (qLMXBs) containing neutron stars (NSs). Quiescent LMXBs are often observed to show a second, harder spectral component generally fit with a PL of photon index $1-2$. This component may make up anywhere from $<5 \%$ to the majority of the detected X-ray flux (Rutledge et al. 2001; Heinke et al. 2003b; Wijnands et al. 2005). We plot the effect of including such a harder PL component (with photon index 1.5), which makes up $20 \%$ of the $0.5-6 \mathrm{keV}$ flux, in Figure 4.

We note immediately that there are very few X-ray sources lying along the track for NS atmospheres in Figure 4. However, there are substantial numbers of sources between this track and the track for NS atmospheres plus a $20 \%$ PL component. Objects with this range of X-ray colors at the extinction of Terzan 5 would require PL indices greater than 3 or MEKAL temperatures less than $2 \mathrm{keV}$. Among the globular clusters for which excellent optical and ACIS data are available (47 Tuc [Grindlay et al. 2001a; Edmonds et al. 2003], NGC 6397 [Grindlay et al. 2001b], $\omega$ Cen [Cool et al. 2002; Gendre et al. 2003], NGC 6752 [Pooley et al. 2002a], M4 [Bassa et al. 2004], NGC 288 [Kong et al. 2006], and M30 [Lugger et al. 2006]), there are no examples of CVs of similar brightness and very soft spectrum, ${ }^{13}$ leading us to suspect that these sources are probably qLMXBs. Several authors have suggested that most globular cluster qLMXBs appear softer than $\mathrm{CVs}$, even allowing for a substantial power-law contribution (e.g., Pooley et al. 2003; Heinke et al. 2003c). Pooley \& Hut (2006) plot a modified XCMD for all globular cluster sources (their Fig. 1), which shows that nearly all identified globular cluster CVs have spectra harder than CX 3 . We note that some bright qLMXBs have relatively hard spectra (see Wijnands et al. 2005 and below) and that there may be large populations of faint qLMXBs with hard spectra (Jonker et al. 2004; Heinke et al. 2005a). However, given studies of other clusters, we think it likely that the X-ray sources we have labeled with open squares in Figure 4 are mostly qLMXBs and that they represent the majority of the qLMXBs in that luminosity range. The high extinction toward Terzan 5 makes spectral analysis of these sources difficult and optical studies with current instrumentation nearly impossible, so it could be a long time before the X-ray sources in Terzan 5 can be conclusively identified.

\subsection{X-Ray Color-Color Plot}

We can also study the $\mathrm{X}$-ray colors of the $\mathrm{X}$-ray sources in Terzan 5 by producing a color-color plot. Our motivation is to compare the X-ray colors of Terzan 5 sources, in relatively hard $\mathrm{X}$-ray bands, to the faint X-ray sources discovered in the Galactic center by Muno et al. (2003). It has been suggested that the relatively bright $\left(L_{\mathrm{X}} \sim 10^{32}-10^{33} \mathrm{ergs} \mathrm{s}^{-1}\right)$ hard X-ray sources in globular clusters are largely composed of "intermediate polars" or DQ Her stars (Grindlay et al. 1995; Edmonds et al. 1999), CVs in which the accretion flow is channeled by the magnetic field of the white dwarf onto its magnetic poles (Patterson \& Raymond 1985). It has also been suggested that the bright hard X-ray sources at the Galactic center are intermediate polars (Muno et al. 2003). We test whether the X-ray sources in these two environments show similar X-ray spectra in a band where interstellar extinction is not very important $(>2 \mathrm{keV})$.

We use the same bands and colors as in Figure 12 of Muno et al. (2003) with colors of the form $(h-s) /(h+s)$, where $h$ and $s$ are the numbers of counts in the harder and softer bands, respectively. The medium color uses the bands 3.3-4.7 and 2.0$3.3 \mathrm{keV}$, while the hard color uses the bands $4.7-8.0$ and 3.3$4.7 \mathrm{keV}$. In Figure 5 we plot the locations of Terzan $5 \mathrm{X}$-ray sources with more than 20 counts and overlay the colors of several spectral models. Since the detectors (ACIS-I vs. ACIS-S) and absorption columns are different, the colors are not directly comparable; thus, it is important to plot colors for various models. Comparison of Figure 5 with Figure 12 of Muno et al. (2003) shows that a photon index of 1-2 is a reasonable description of the majority of the Terzan 5 X-ray sources, while the Galactic center sources are better described by a median photon index near 0 . A portion of the Galactic center sources are even harder. This indicates that the Galactic center sources may be substantially different from the hard sources in Terzan 5. (This question will be investigated in more detail in a forthcoming paper.) We note that

\footnotetext{
${ }^{13} \mathrm{X} 10$ in 47 Tuc is the softest bright $\mathrm{CV}$ we know of, but it still has a powerlaw photon index less than 3 .
} 


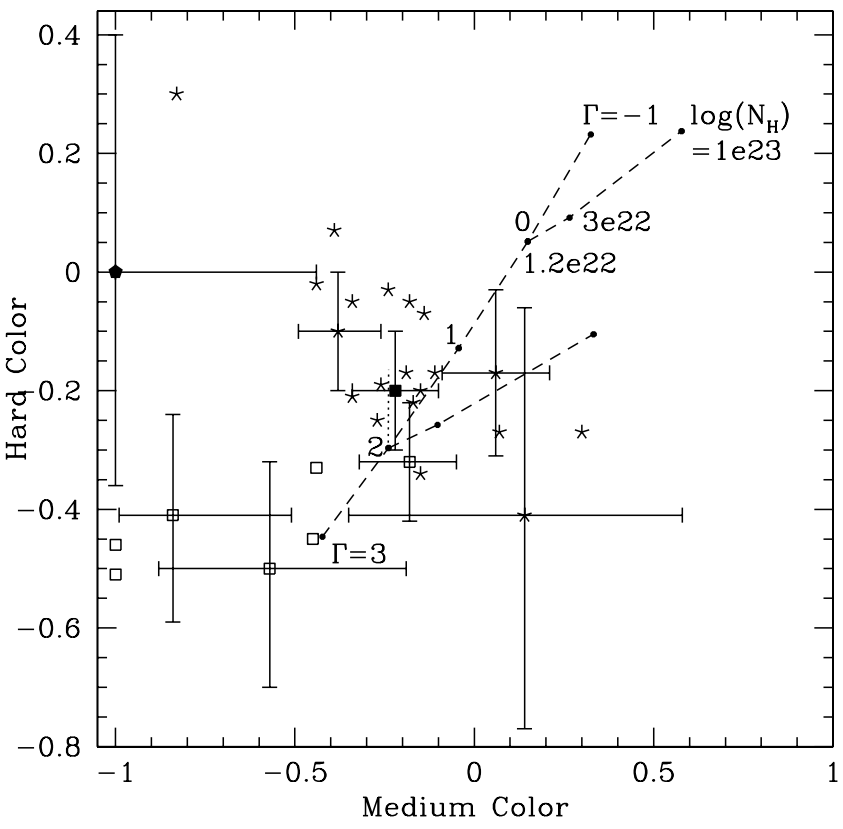

FIG. 5.-X-ray color-color plot for Terzan 5, showing sources associated with the cluster and having more than 20 counts. The dashed tracks indicate colors for a power law of different spectral indices, and (shifting to right) for PL models of fixed spectral index with increasing $N_{\mathrm{H}}$. The dotted line ascending from the $\Gamma=2$ power-law model indicates the impact of adding a $6.5 \mathrm{keV}$ Fe line of up to $1.6 \mathrm{keV}$ equivalent width. See the text for definitions of colors. Symbols are the same as in Fig. 4. [See the electronic edition of the Journal for a color version of this figure.]

one very hard bright source (photon index $=0.2$ ) has been identified in the globular cluster Terzan 1 (Cackett et al. 2006).

\subsection{Spectral Fitting and Time Variability}

We extracted spectra and associated files for the brighter cluster sources as described in $\S 2.1$ above. We performed spec- tral fitting for sources with more than 60 counts (Table 3 ). To allow fitting with faint sources, we perform binning using 10 counts $\mathrm{bin}^{-1}$ for sources with more than 70 counts and 8 counts bin ${ }^{-1}$ for four sources with $\sim 60-70$ counts. We also performed spectral fits with 20 or 5 counts bin $^{-1}$, using the $C$-statistic instead of the $\chi^{2}$ statistic. We found that the results from each method were comparable, with our preferred binning giving slightly tighter constraints in several cases.

We choose three models designed to cover the range of spectra typically seen in globular cluster X-ray sources in this luminosity range, all absorbed by $N_{\mathrm{H}} \geq 1.2 \times 10^{22} \mathrm{~cm}^{-2}$ and a dust column appropriate for $A_{V}=6.7$. For the latter we use the scatter XSPEC code kindly provided by P. Predehl (Predehl et al. 2003). Our continuum models include a thermal plasma MEKAL spectrum (which can be physically appropriate for CVs or ABs), a simple power law (physically appropriate for synchrotron radiation from bright MSPs), and a two-component model consisting of a hydrogen-atmosphere NS model (Heinke et al. 2006) plus a power law (physically appropriate for qLMXBs). To permit interesting constraints on some parameters for this model, we fix the NS radius $(10 \mathrm{~km})$, mass $\left(1.4 M_{\odot}\right)$, and distance $(8.7 \mathrm{kpc})$; we also fix the slope of the associated PL model to $\Gamma=1.5$, a typical slope for hard power-law components in qLMXB spectra (Rutledge et al. 2001).

We find, in contrast to other globular clusters, that none of the bright sources are spectrally consistent with a simple hydrogenatmosphere model. Some relatively soft sources, with effective PL photon indices greater than 2, can be modeled as the combination of a hydrogen-atmosphere model and a harder PL component, as often seen in Galactic qLMXBs (e.g., Campana et al. 1998; Rutledge et al. 2001). This is counter to the suggestion by Heinke et al. (2003c) that globular cluster qLMXBs do not possess this harder component unless they have recently been active, as there is no evidence for outbursts by more than one LMXB in Terzan 5 in the past $30 \mathrm{yr}$ (but see Wijnands et al. 2005 for a discussion).

TABLE 3

Spectral Fits to Brighter Terzan 5 Sources

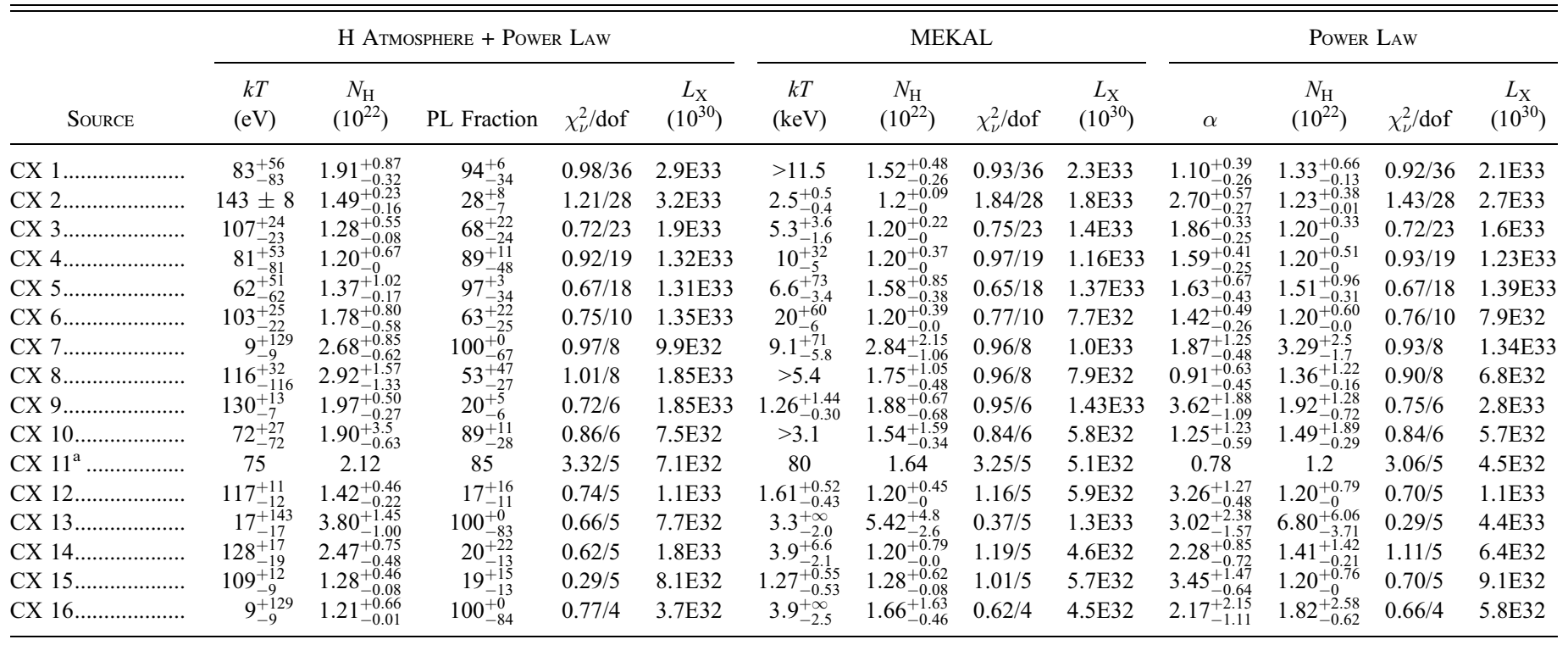

NoTES.-Spectral fits to cluster sources, with background subtraction, in XSPEC. Errors are $90 \%$ confidence for a single parameter; spectra are binned with 10 counts $\mathrm{bin}^{-1}$ for all sources with more than 70 counts, 8 counts bin ${ }^{-1}$ for sources with fewer counts. All fits include photoelectric absorption forced to be $\geq 1.2 \times 10^{22} \mathrm{~cm}^{-2}$, the cluster $N_{\mathrm{H}}$ derived from near-infrared studies (Cohn et al. 2002), plus dust scattering for an assumed $A_{V}=6.7$. For hydrogen atmosphere plus power-law fits, we fixed the power-law photon index to 1.5 , the NS radius to $10 \mathrm{~km}$, and the NS mass to $1.4 M_{\odot}$.

${ }^{\mathrm{a}} \mathrm{CX} 11$ 's spectrum does not produce acceptable fits with any of these models. 


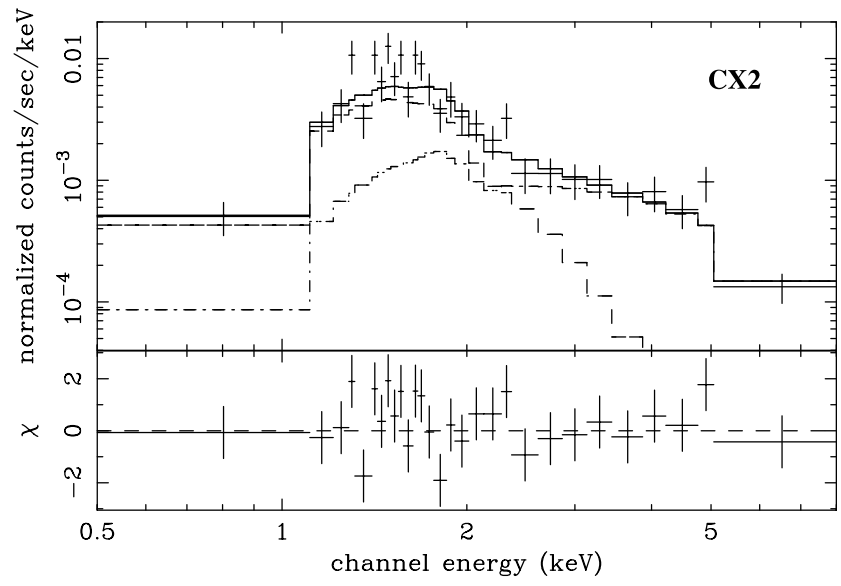

FIG. 6.-X-ray spectrum of CX 2, showing the data (crosses), the model (solid line), and the portion of the model due to each component (dashed line, hydrogen atmosphere model; dot-dashed line, power-law component).

We performed a Kolmogorov-Smirnov (K-S) test for variability (implemented in ACIS_EXTRACT) on the event files for each source associated with Terzan 5 . Those nine sources that indicate variability with $>95 \%$ confidence are indicated in Table 1 with "V?"; the three sources that have $>99 \%$ confidence are indicated with "V." Since we are testing 50 sources, we might expect that two sources might be spuriously identified as variable for a confidence limit of $95 \%$; on the other hand, faint sources are unlikely to be identified as variable, so the above estimate is overly conservative.

Some sources can be identified as variable between the 2000 and 2002 observations of Terzan 5 and are indicated with " $Y$ " in Table 1. For those sources that were detected in both 2000 and 2003, we have extracted spectra from the 2000 observation to test whether the sources require variability. We reprocessed the 2000 data in the same manner as the 2003 data. We extracted spectra from $1^{\prime \prime}$ circles and extracted background for most sources from 2".5 annuli around these circles that do not overlap other sources (for a few sources we carefully chose alternate background regions). Spectra were grouped with 10 or 20 counts per bin. We note that the data quality in 2000 is much poorer, due to the high background from the transient outburst. For bright 2003 sources not detected in 2000, we followed the procedure of Heinke et al. (2005b; see also Muno et al. 2003) to identify variability at the $3 \sigma$ level from nondetections.

\subsection{Discussions of Individual Sources}

\subsubsection{EXO 1745-248 = CXOGlb J174805.2-244647 = CX 3}

This source, the quiescent counterpart to the bright transient LMXB, has already been discussed by Wijnands et al. (2005). Our analysis agrees that the spectrum is dominated by a hard PL component. In addition to the models in Table 3, we fit a NS + PL model in which the PL index was allowed to vary. This model produced a NS temperature of $91_{-91}^{+40} \mathrm{eV}$, giving the NS component $133_{-13}^{+33} \%$ of the total unabsorbed $0.5-10 \mathrm{keV}$ flux.

CX 3 is not identified as clearly variable during the 2003 observation, with a K-S probability of nonvariance of $6.6 \%$. This finding differs from that of Wijnands et al. (2005; less than 5\% probability of nonvariance), but this small difference is likely due to different (but equally justifiable) choices of data reduction procedures.

$$
\text { 3.5.2. CX 2 = CXOGlb J174805.4-244637 =W3 }
$$

This source, observed by Heinke et al. (2003a) and labeled W3, is the second brightest source in Terzan 5. Its spectrum (Fig. 6) is the clearest example of a two-component spectrum among our sources. Adding a NS atmosphere component to an absorbed PL fit gives an $F$-test probability of $3.3 \%$ that the improvement in statistic could have arisen by chance. We regard this source as an almost certain qLMXB.

It is clear from the recorded counts and inferred X-ray luminosities in Table 1 compared to Heinke et al. (2003a) that CX 2 has varied in brightness between 2000 and 2003 . We confirm this by simultaneously fitting spectra of CX 2 from 2000 and 2003. CX 2's 2000 flux was at $26 \% \pm 7 \%$ of its 2003 flux, determined from scaling a PL fit. For our preferred NS atmosphere plus PL model, acceptable fits (null hypothesis probabilities [nhp] $>10 \%$ ) can be obtained by varying the NS atmosphere temperature, varying the PL normalization, or varying the $N_{\mathrm{H}}$ column, while keeping the other parameters fixed. CX 2 did not demonstrate clear variability during the 2003 observation.

$$
\text { 3.5.3. CX 20= CXOGlb J174803.0-244640 }
$$

This source has an unusually soft spectrum, with substantial flux below $1 \mathrm{keV}$. Our method of computing X-ray luminosity, based on an assumption of $N_{\mathrm{H}}=1.2 \times 10^{22} \mathrm{~cm}^{-2}$, assigns a very high $0.5-1.0 \mathrm{keV}$ (and thus $0.5-2.5 \mathrm{keV}$ ) luminosity to this object due to these soft photons. However, our spectral analysis of this source reveals that no continuum spectral model can reproduce the spectral shape if the cluster $N_{\mathrm{H}}$ is assumed. We find a $N_{\mathrm{H}}$ best fit at $6 \times 10^{20} \mathrm{~cm}^{-2}$ for either MEKAL or PL models, with the $90 \%$ confidence upper limit of $3 \times 10^{21}$ or $5 \times 10^{21} \mathrm{~cm}^{-2}$, respectively. This causes us to suggest that CX 20 may be a foreground star.

Several additional pieces of information support our assertion. We note that this source lies outside the cluster core (see Fig. 1), as is likely for a randomly placed foreground object. CX 20 displays strong variability, with 25 of 40 photons being received within $2.3 \mathrm{ks}$ (of $35.3 \mathrm{ks}$ total). Our K-S test for variability gives a $\left(5 \times 10^{-4}\right) \%$ probability of such a distribution by chance. Such flaring behavior suggests a stellar flare of a coronally active star.

A possible optical counterpart appears on the SuperCOSMOS UKST blue $\left(B_{j}\right)$ plates available through the Aladin ${ }^{14}$ image server. The USNO-B1.0 catalog (Monet et al. 2003) identifies a nonstellar object (presumably due to crowding) at $17^{\mathrm{h}} 48^{\mathrm{m}} 03^{\mathrm{s}} .04$, $-24^{\circ} 46^{\prime} 40^{\prime \prime} 9$ (quoted position errors 0."5), with $R=12.57$ and $B=18.62$. This position is consistent with CX 20 within the USNO errors. However, the color of the star is too red to be consistent with a foreground star at the $N_{\mathrm{H}}$ measured above, so unless the USNO magnitudes are in error due to crowding, this star is unlikely to be the stellar counterpart.

\subsubsection{Other Potential qLMXBs}

The following sources have rather soft X-ray colors, indicating effective PL photon indices larger than 3. A PL photon index larger than 3 is rarely observed among non-qLMXBs in this $L_{X}$ range; a likely physical explanation is the presence of a blackbody-like NS atmosphere component.

$C X 9=$ CXOGlb J174804.8-244644 =W4.- This source exhibits a soft spectrum, with an inferred PL photon index of $3.62_{-1.09}^{+1.88}$. The steep spectrum suggests that it is dominated by a NS surface, but an absorbed NS atmosphere model alone produces a relatively poor fit (nhp $=7 \%$ ) with clear residuals above $3 \mathrm{keV}$. Adding a NS atmosphere component to a PL fit allows the power law to be less steep (best fit $\Gamma=2.2$ ), but an $F$-test does not indicate that the NS component is required to improve the fit.

14 See http://aladin.u-strasbg.fr/aladin.gml. 
We designate this source as a possible qLMXB. Simultaneous fits to the 2000 and 2003 spectra do not require variability.

CX $12=$ CXOGlb J174806.2-244642 =W2.-This source shows an inferred PL photon index of $3.26_{-0.48}^{+1.27}$, also suggesting a NS surface. Like CX 9, a NS atmosphere alone is a poor fit (nhp $=1 \%)$, and adding a NS atmosphere component does not substantially improve the PL fit, although it allows a less steep power law. We designate CX 12 as another possible qLMXB.

Simultaneous fits to the 2000 and 2003 spectra suggest that CX 12 may have been fainter in 2000 (best fit gives 2000 flux at $42 \%$ of 2003 flux), but the errors are large enough that variability is not required at $90 \%$ confidence.

CX $14=$ CXOGlb J174805.3-244652.- This source shows an inferred PL photon index of $2.28_{-0.72}^{+0.85}$, perhaps not as soft as other suggested qLMXBs. A NS atmosphere alone is again a poor fit, but adding a NS component does improve the PL fit (an $F$-test gives an $8.5 \%$ probability that this improvement is due to chance). This is a marginal candidate for a qLMXB.

CX 14's location 6" from EXO 1745-248 during its 2000 outburst prevents measurement of possible long-term variability. We identify variability, with $98.2 \%$ confidence, during the 2003 observation.

CX 15 = CXOGlb J174804.1-244647 = W8. - This source shows an inferred PL photon index of $3.45_{-0.64}^{+1.47}$, suggesting a NS component. Again, a NS atmosphere alone is a poor fit, but adding a NS component improves a PL fit (an $F$-test gives a $6.8 \%$ probability of such a chance improvement). CX 15 is a good candidate for a qLMXB. Simultaneous fits to 2000 and 2003 data reveal no evidence for variability.

The above sources are reasonable candidates for qLMXBs. Several other X-ray sources are softer than these sources but have fewer than 60 counts, making spectral fitting difficult. We think these softer sources are reasonable candidates for qLMXBs, probably with smaller PL spectral components (see Fig. 4). The level of certainty in classification of qLMXBs that can be attained in other clusters has not been reached for these sources, with the exception of CX 3 and CX 2. In addition, the luminosities of these candidate qLMXBs must be regarded as extremely poorly determined, since their inferred luminosities depend on extrapolation from a few counts below $1.5 \mathrm{keV}$. This makes comparison of the source content of Terzan 5 with other clusters rather uncertain.

\subsubsection{Other Sources Seen in 2000}

A number of X-ray sources appear to be fainter in 2000 than in 2003, as determined from fitting an absorbed PL model to both spectra, with only a normalization constant allowed to vary between the two spectral fits. For CX 6 (W5), the normalization of the 2000 data is $33_{-33}^{+44} \%$ that of the 2003 data. For CX 7 (W9), the 2000 normalization is $30_{-30}^{+51} \%$ of the 2003 normalization. For CX 11 (W7), the 2000 normalization is consistent $\left(79_{-69}^{+79} \%\right.$ ) with that of 2003, as well as for CX 16 (W10, 122 $2_{-91}^{+104} \%$ ). Only for CX 8 (W6) is the best-fit normalization of the 2000 data marginally higher $\left(1.5_{-0.5}^{+0.6}\right)$ than the 2003 data. We find it rather odd that so many sources were apparently fainter in 2000 than in 2003. We can rule out possibilities such as incorrect exposure times or oversubtraction of background. There are roughly twice as many sources with $L_{\mathrm{X}}>10^{32} \mathrm{ergs} \mathrm{s}^{-1}$ as inferred from incompleteness tests on the 2000 data, which we also do not fully understand.

\subsubsection{New Sources}

Two bright sources are apparent in the 2003 data that would have been clearly detected at that brightness in 2000 . The brightest source in our observation, CX 1 (CXOGlb J174804.5-244641), has a $3 \sigma$ upper limit of $6.47 \times 10^{32}$ in the 2000 observation, a factor of 5 lower than in its 2003 detection. CX 1 has a rather hard spectrum, with $\Gamma=1.10_{-0.26}^{+0.39}$, and an unusually high luminosity of $L_{\mathrm{X}}=3.7 \times 10^{33} \mathrm{ergs} \mathrm{s}^{-1}$. CX 4 (CXOGlb J174804.7-244708) has a $3 \sigma$ upper limit of $2.73 \times 10^{32}$ in the 2000 observation, also a factor of 5 below its 2003 detection. Its spectrum is slightly softer than CX $1\left(\Gamma=1.59_{-0.25}^{+0.41}\right)$. Fainter new 2003 sources cannot be ruled out in the 2000 data, and readers might persuade themselves that they see evidence for, e.g., CX 10, CX 13, and CX 19 in the 2000 data.

\subsubsection{Millisecond Pulsars?}

Ransom et al. (2005) have identified a large population of radio MSPs in Terzan 5, of which some may be detectable X-ray sources. A typical soft-spectrum low-luminosity ( $\mathrm{few} \times 10^{30} \mathrm{ergs}$ $\mathrm{s}^{-1}$ ) MSP like those in 47 Tuc (Grindlay et al. 2002; Bogdanov et al. 2006) would contribute fewer than 0.5 counts to our data set. Positions for Terzan 5 A and C (Fruchter \& Goss 2000) show no $0.5-7 \mathrm{keV}$ counts, allowing $95 \%$ confidence upper limits on their flux of (1-3) $\times 10^{31} \mathrm{ergs} \mathrm{s}^{-1}$, depending on the chosen spectrum. However, some MSPs are brighter with harder spectra, typically those with higher spin-down luminosities (e.g., PSR 1821-24 in M28; Becker et al. 2003) or those that show hard spectra from shocks between the pulsar wind and material from the companion (e.g., 47 Tuc W [Bogdanov et al. 2005] and NGC 6397 A [Grindlay et al. 2002]). Some of these MSPs should be detectable in Terzan 5. A preliminary position for one MSP is indeed a very close match to the position of the hard X-ray source CX 10 (S. Ransom 2005, private communication). Other X-ray sources may be identified with MSPs as more positions become available.

\subsection{Spatial Distribution of X-Ray Sources}

We estimate the ratio of the masses of the X-ray binaries in Terzan 5 to the masses of stars in Terzan 5 , by comparing their radial distribution to the radial distribution of cluster stars. We use the method described by Heinke et al. (2003c; following Grindlay et al. 1984) to fit the distribution of X-ray sources with a generalized King model, of the form

$$
S(r)=S_{0}\left[1+\left(r / r_{c *}\right)^{2}\right]^{(1-3 q) / 2},
$$

where $r_{c *}$ is the optical core radius of the cluster and $q$ is the ratio of the masses of the X-ray sources and the stars that define the cluster core radius.

We use the distribution of 40 cluster sources above 10 counts (below which we are probably substantially incomplete in the core) to measure the radial profile of Terzan $5 \mathrm{X}$-ray sources. We assume (based on our analysis of X-ray sources across the S3 chip, above in $\S 2.1$ ) that one of these sources is a background (or foreground) source. A maximum likelihood fit to the radial profile with our model gives a good fit, with a mass ratio $q=1.43 \pm$ 0.11 (Fig. 7). This can be compared with the value of $q=1.63 \pm$ 0.11 found by Heinke et al. (2005b) for 47 Tuc. It is possible that incompleteness in the core may affect our result, but increasing the cutoff value from 10 to $20(q=1.45 \pm 0.14)$ or $40(q=$ $1.53 \pm 0.18)$ counts does not significantly alter the inferred value of $q$.

For an assumed turnoff mass of $0.9 M_{\odot}$ (e.g., Bergbusch \& Van den Berg 2001), we find a characteristic Terzan 5 X-ray source mass of $1.29 \pm 0.10 M_{\odot}$. For the subsample of 11 likely qLMXBs plus the known transient LMXB, we derive $q=1.64 \pm 0.25$, not substantially different from the result for the entire sample but consistent with neutron stars and low-mass companions. We 


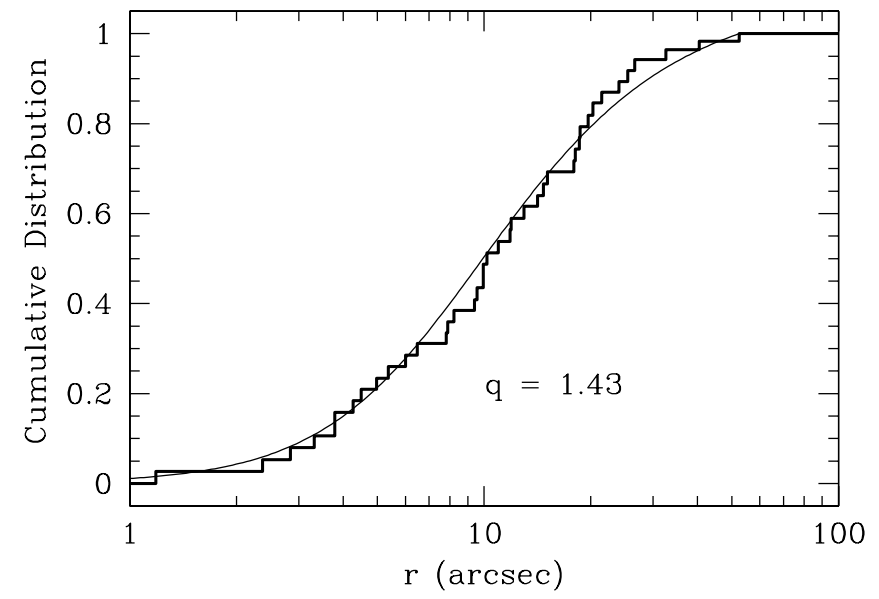

FIG. 7.-Cumulative radial profile of Terzan 5 X-ray sources with $>10$ counts, fitted with our maximum likelihood generalized King profile.

also note that our lower sensitivity to soft sources (due to extinction) may cause us to miss some qLMXBs. Due to the relatively high mass of NSs compared to white dwarfs and cluster stars, this effect may bias our $q$ for the total sample downward, compared to relatively unabsorbed clusters.

\subsection{Luminosity Function and Unresolved Sources}

We choose a limiting luminosity of $L_{\mathrm{X}}(1-6 \mathrm{keV}) \sim 10^{32}$ ergs $\mathrm{s}^{-1}$ for the following analyses. For hard sources this is $\sim 15$ counts, a limit to which we are easily complete. For soft sources the limit is more uncertain. For the NSATMOS hydrogen atmosphere model alone, a 5 count detection is $L_{X}=1.3 \times 10^{32} \mathrm{ergs} \mathrm{s}^{-1}$. However, we have found that few if any sources in Terzan 5 are well described by such a model, most requiring a harder power-law component. Adding a 20\% power-law component gives 8 counts total, roughly our completeness limit. We use a limiting luminosity of $10^{32} \mathrm{ergs} \mathrm{s}^{-1}$ but recognize that our results may be biased by the loss of soft sources (which is difficult to quantify).

We compute an X-ray luminosity function (XLF) for our sources above this limit of the form $(N>L) \propto L^{-\alpha}$ (following Johnston $\&$ Verbunt 1996). We find a best-fit slope $\alpha$ of $0.71_{-0.21}^{+0.25}$ (errors indicate where the K-S probability falls below $10 \%$ ); if using the $0.5-2.5 \mathrm{keV}$ luminosities (and a limit of $5 \times 10^{31} \mathrm{ergs} \mathrm{s}^{-1}$ ), we find $\alpha=0.44 \pm 0.03$. We note that the $0.5-2.5 \mathrm{keV}$ slope is consistent with the XLF slope found by Pooley et al. (2002b) for a similar luminosity range in NGC 6440 by the same method, although our $0.5-2.5 \mathrm{keV}$ luminosities are rather uncertain.

The X-ray luminosity from unresolved sources can be constrained, once the background and the "spill" from the PSF wings of identified sources are subtracted. We measured the counts outside the source extraction regions within the core and halfmass regions in seven energy bands and subtracted the average background (measured in a large area with no bright sources west of the cluster) and the appropriate "spill" from known sources in each band. We detect a signal above background in each band above $1 \mathrm{keV}$ and below $4.5 \mathrm{keV}$. We find a total of $96 \pm 19$ counts from unresolved sources in the core and $429 \pm$ 49 counts from unresolved sources within the half-mass radius. This translates (using our method in $\S 2$ of computing conversions for each band) to $L_{\mathrm{X}}(1-4.5 \mathrm{keV})=4.3 \pm 0.9 \times 10^{32} \mathrm{ergs}$ $\mathrm{s}^{-1}$ for the core and $L_{\mathrm{X}}(1-4.5 \mathrm{keV})=1.8 \pm 0.2 \times 10^{33} \mathrm{ergs} \mathrm{s}^{-1}$ for unresolved sources within the half-mass radius. If we assume a $7 \mathrm{keV}$ bremsstrahlung spectrum, $L_{\mathrm{X}}(1.0-6 \mathrm{keV}) \sim 2.2 \times 10^{33}$ for the half-mass radius. We can constrain the $1-6 \mathrm{keV}$ XLF by comparing the total detected and undetected sources with $L_{\mathrm{X}}<$ $10^{32}$ ergs s$^{-1}$ (total $1-6 \mathrm{keV}_{\mathrm{X}}=3.2 \times 10^{33} \mathrm{ergs} \mathrm{s}^{-1}$ ) to those with $10^{32} \operatorname{ergs~s}^{-1}<L_{\mathrm{X}}(1-6 \mathrm{keV})<10^{33} \mathrm{ergs} \mathrm{s}^{-1}$ (total $L_{\mathrm{X}}=$ $8.4 \times 10^{33} \mathrm{ergs} \mathrm{s}^{-1}$ ). This ratio suggests an XLF index of $\alpha=$ 0.44 in the $1-6 \mathrm{keV}$ band, somewhat less than inferred above.

\section{COMPARISONS WITH OTHER CLUSTERS}

Terzan 5 has the largest observed number of X-ray binaries above $L_{X}=10^{32} \mathrm{ergs} \mathrm{s}^{-1}$ of any globular cluster in the Galaxy. With $28 \mathrm{X}$-ray sources having inferred unabsorbed X-ray luminosities $(0.5-2.5 \mathrm{keV})>10^{32} \mathrm{ergs} \mathrm{s}^{-1}$ (or 33 , for the $1-6 \mathrm{keV}$ range), Terzan 5 contains more than twice as many X-ray binaries in this luminosity range as NGC 6440 and NGC 6266, the next richest X-ray clusters studied so far (Pooley et al. 2002b, 2003). By comparing results from study of this cluster to results from other clusters, we can test the dependence of X-ray source production on cluster properties, such as the central density of the cluster.

\subsection{Dependence of Encounter Frequency on $\rho, r_{c}$, and Metallicity}

Verbunt \& Hut (1987) parameterized the production rate of $\mathrm{X}$-ray binaries in globular clusters as proportional to the square of the central density $\rho$ and the volume of the core (where most interactions take place) $\sim r_{c}^{3}$ while inversely proportional to the velocity dispersion in the core. Thus, $\Gamma \propto \rho^{2} r_{c}^{3} / \sigma$. For a King model globular cluster (and for any virialized cluster), the central velocity dispersion should be proportional to $\rho^{0.5} r_{c}$, leading to $\Gamma \propto \rho^{1.5} r_{c}^{2}$.

We use the system of Heinke et al. (2003c; see Johnston \& Verbunt 1996) to parameterize the dependence of X-ray binary production on cluster properties. This system compares the distribution of X-ray binaries across a number of clusters with the distribution of production rate for given dependences of $\Gamma$ on cluster parameters. We use $\Gamma \propto \rho^{\alpha} r_{c}^{\beta}\left(Z / Z_{\odot}\right)^{\delta}$, with $\rho$ the central luminosity density, $r_{c}$ the core radius, ${ }^{15}$ and $Z / Z_{\odot}$ the cluster metallicity. We add dependence of $\Gamma$ on metallicity, as indicated by studies of LMXBs in globular cluster systems of elliptical galaxies (Kundu et al. 2002; Jordán et al. 2004). The most detailed such study (Jordán et al. 2004) found a dependence $\Gamma \propto \rho^{1.08 \pm 0.11} r_{c}^{2}\left(Z / Z_{\odot}\right)^{0.33 \pm 0.1}$ for production of LMXBs in the clusters of M87.

This analysis generally follows that of Heinke et al. (2003c) differing in three respects: We study the effect of metallicity in addition to central density and core radius (for most clusters we use the values from the Harris [1996] revision of 2003, otherwise using values from Heinke et al. [2003c]). We update numbers of likely qLMXBs and hard sources (bright and faint) for several clusters, these being Terzan 5 (this paper), NGC 6266 (unpublished work by the authors; we use five qLMXBs, seven hard sources with $L_{\mathrm{X}}>10^{32} \mathrm{ergs} \mathrm{s}^{-1}$, and 21 hard sources with $10^{32}$ ergs s$^{-1}>L_{\mathrm{X}}>10^{31} \mathrm{ergs} \mathrm{s}^{-1}$ ), 47 Tuc (Heinke et al. 2005b), and NGC 6397 (changing the distance from 2.7 to $2.5 \mathrm{kpc}$, in accord with Gratton et al. 2003). We use the same numbers as Heinke et al. (2003c) for the clusters M80, M28, NGC 6752, $\omega$ Cen, M30, NGC 5904 (M5), M22, M13, NGC 6121 (M4), and NGC 6366. Finally, we make two changes to the code computing the K-S probabilities. One change corrects a coding error in the program of Heinke et al. (2003c) that decreased the best-fit densities for qLMXBs and bright CVs (see below). The other

\footnotetext{
15 This is a somewhat simplistic way of treating core-collapsed clusters, which have more complex radial structures and may have a more complex binary history; we defer a more sophisticated analysis.
} 

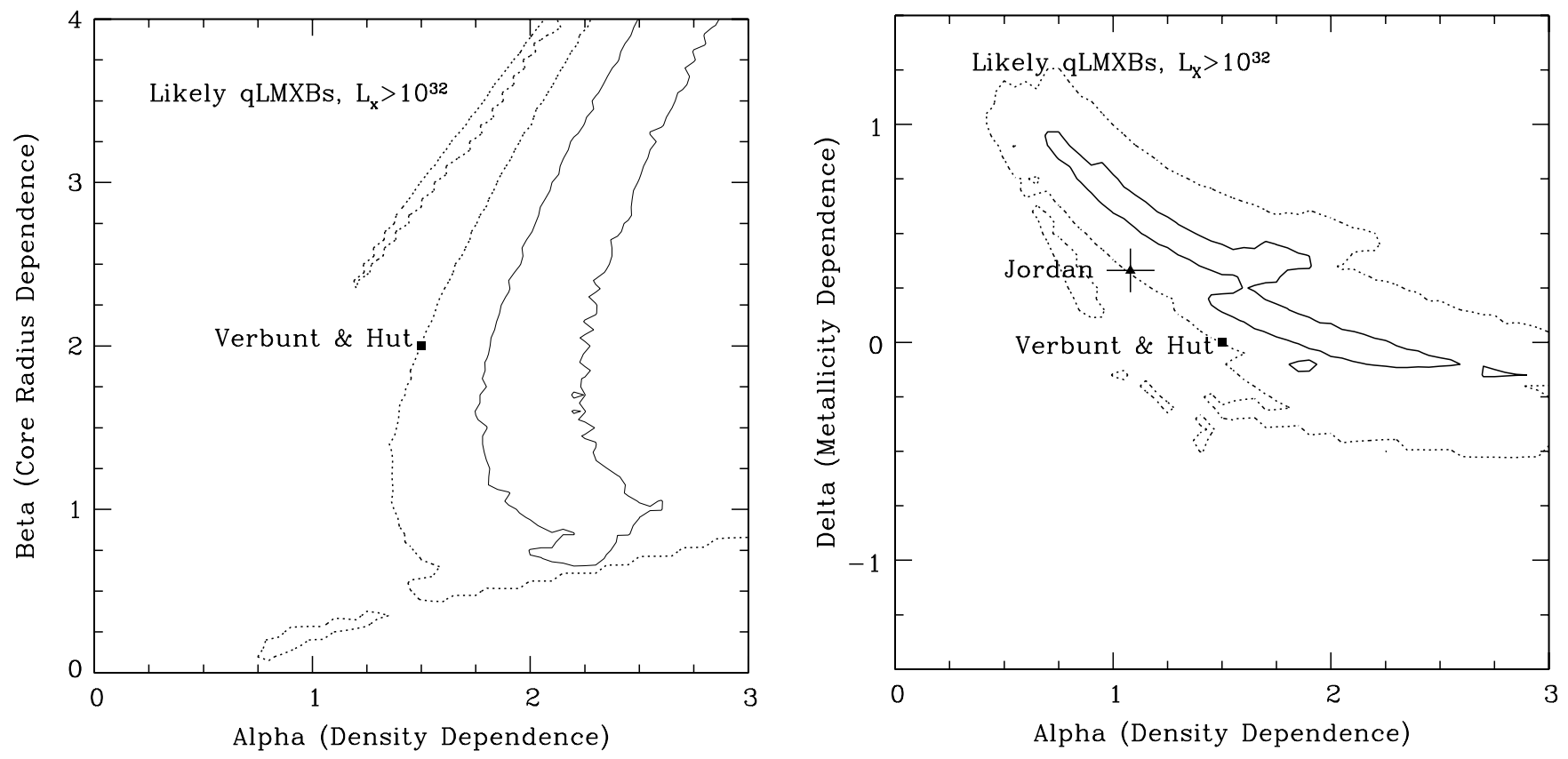

FIG. 8.- Contours of the K-S probability for different parameterizations of the production rate for likely qLMXBs. Left, Dependence of production rate on core density vs. core radius; right, dependence of production rate on core density vs. cluster metallicity. Solid contours enclose $>50 \%$ K-S probability; dotted contours enclose $>10 \%$ K-S probability.

changes an assumption about the distribution of sources within each cluster. The code distributes the sources along a fictional line segment, in which each cluster occupies a length equal to its $\Gamma$, and the clusters are arranged in order of decreasing $\Gamma$. The output is the K-S probability of finding such a distribution of sources along the line if the sources were distributed randomly (with an equal probability of source per unit $\Gamma$ ), thus a measure of the appropriateness of that choice of $\Gamma$. The previous code distributed sources within a cluster evenly within its segment, artificially increasing the K-S probability of that choice of $\Gamma$. This change randomizes the distribution of sources within the cluster segment, effectively decreasing all K-S probabilities.

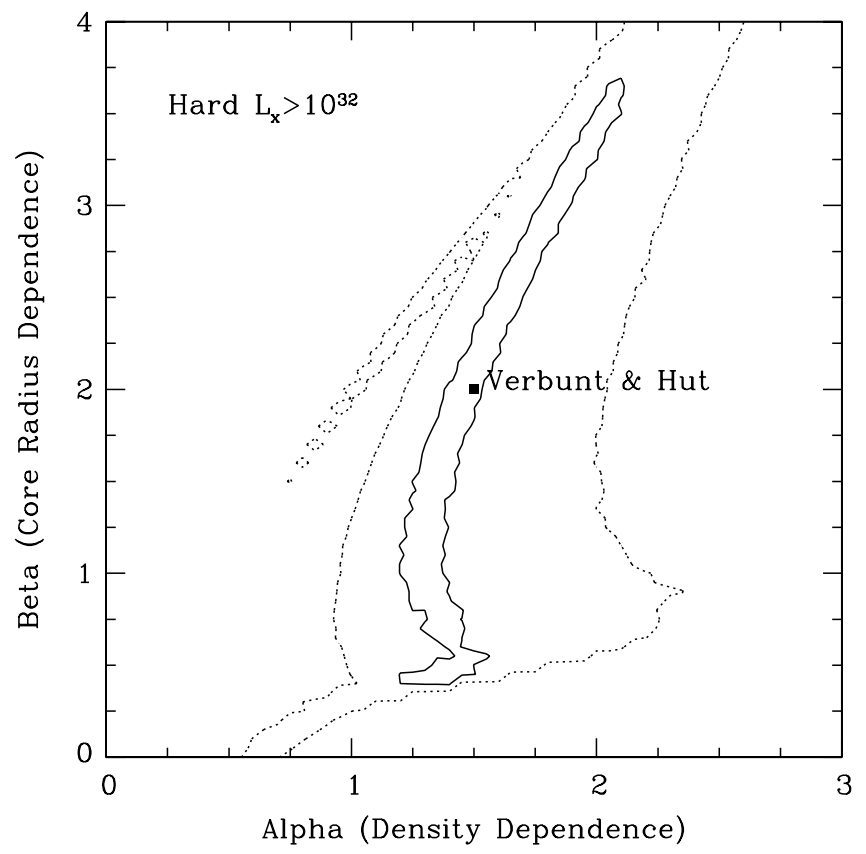

We first test a range of values for $\alpha$ and $\beta$ to explain the distribution of each group of X-ray binaries: qLMXBs [limited to those with $L_{\mathrm{X}}(0.5-2.5 \mathrm{keV})>10^{32} \mathrm{ergs} \mathrm{s}^{-1}$ to reduce incompleteness effects], bright hard sources $\left[L_{\mathrm{X}}(0.5-2.5 \mathrm{keV})>10^{32} \mathrm{ergs}\right.$ $\left.\mathrm{s}^{-1}\right]$, and faint hard sources $\left[L_{X}(0.5-2.5 \mathrm{keV})=10^{31}-10^{32}\right.$ ergs $\mathrm{s}^{-1}$. For each choice of $\alpha$ and $\beta$ we apply a Kolmogorov-Smirnov test (implemented via Press et al. 1992), comparing the distribution of observed sources with a distribution uniform in $\rho^{\alpha} r_{c}^{\beta}$. We plot contours at $50 \%$ and $10 \% \mathrm{~K}-\mathrm{S}$ probabilities in the left panels of Figures 8, 9, and 10.

The likely qLMXBs are barely consistent with the Verbunt \& Hut predictions at the $10 \%$ probability level. The hard bright sources

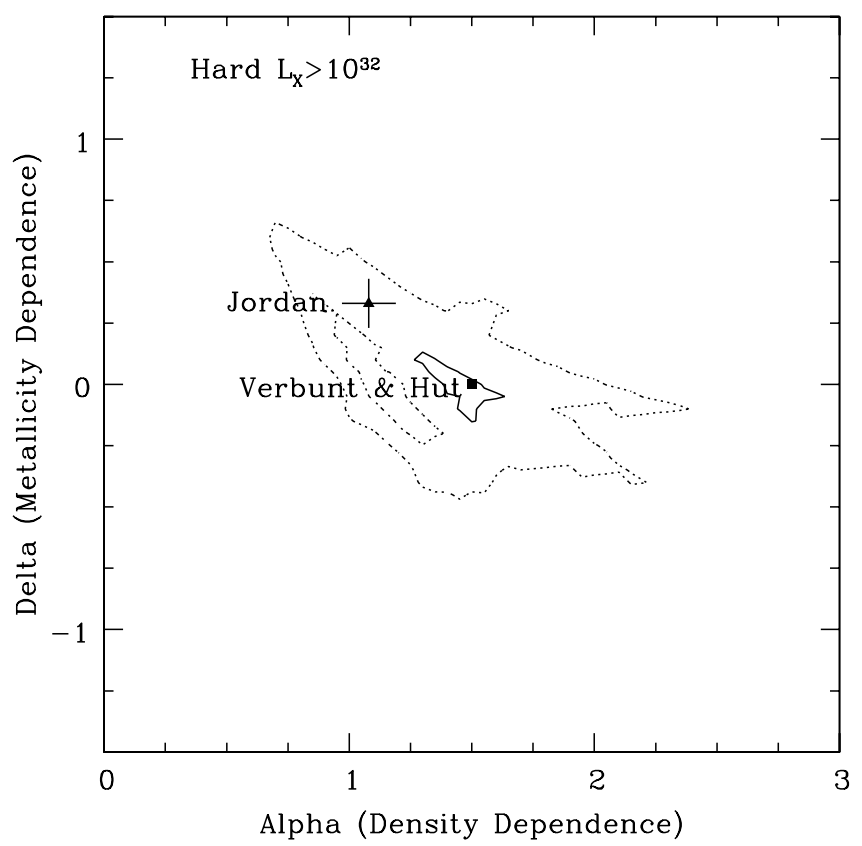

FIG. 9.-Same as Fig. 8, but for hard sources with $L_{\mathrm{X}}>10^{32} \mathrm{ergs} \mathrm{s}^{-1}$ (which may be dominated by bright CVs). 

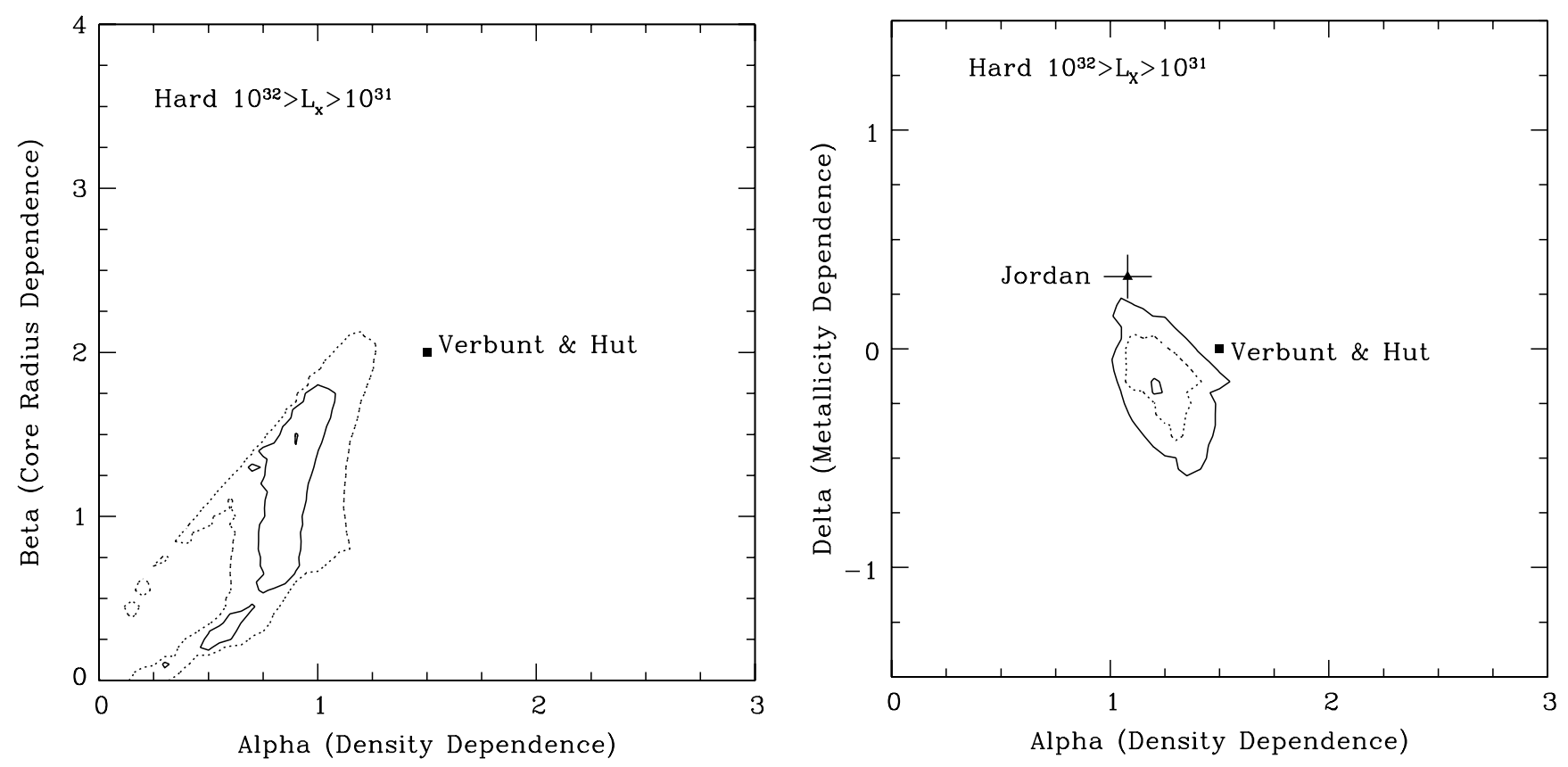

FIG. 10.- Same as Fig. 8, but for hard sources with $10^{32} \mathrm{ergs} \mathrm{s}^{-1}>L_{\mathrm{X}}>10^{31} \mathrm{ergs} \mathrm{s}^{-1}$ (which may be dominated by faint CVs). In the right panel (metallicity vs. density) we have also indicated a contour of $1 \% \mathrm{~K}-\mathrm{S}$ probability with a solid line.

are also consistent with the Verbunt \& Hut predictions, while the fainter hard sources are clearly inconsistent with the Verbunt $\&$ Hut predictions, indicating a lower density dependence. This result contradicts Heinke et al. (2003c) in that it finds a distinction between the distributions of bright hard sources and faint hard sources.

To study the effect of metallicity, we assume $\beta=2$ (thus $\Gamma \propto$ $r_{c}^{2}$ ) and test choices of $\alpha$ and $\delta$ (producing Figs. 8-10, right). The densest clusters studied also tend to be the most metal-rich, so some degeneracy between density and metallicity is seen in these plots. The likely qLMXBs are consistent at the $10 \%$ confidence level with either the Verbunt \& Hut dependence on density and radius or the Jordan et al. dependence. The brighter hard sources are also quite consistent with either of the suggested dependences. However, the fainter hard sources are clearly inconsistent with either of the suggested dependences. In Figure 10 we have plotted, in addition to the contours of $10 \%$ and $50 \% \mathrm{~K}-\mathrm{S}$ probability, the $1 \%$ K-S probability. Neither of the two suggested dependences describe the observations of the faint hard sources, which require a lower dependence on either density or metallicity.

\section{DISCUSSION}

We have not yet identified a clear metallicity dependence in the distribution of qLMXBs and bright CVs in globular clusters. However, considering the evidence for a metallicity dependence in bright LMXBs in extragalactic globular clusters by Kundu et al. (2002) and Jordán et al. (2004) we think it likely that additional observations of Galactic globular clusters will show increasing evidence for a clear dependence. From the information we have, it seems that fainter hard X-ray sources $\left(10^{31} \mathrm{ergs} \mathrm{s}^{-1}<L_{\mathrm{X}}<\right.$ $10^{32} \mathrm{ergs} \mathrm{s}^{-1}$ ) do not show a metallicity dependence of the strength observed for bright LMXBs by Jordán et al.

One important result from this study is the identification of a possible difference in the distribution among globular clusters of the brighter and fainter hard X-ray sources, above and below $L_{\mathrm{X}}(0.5-2.5 \mathrm{keV})=10^{32} \mathrm{ergs} \mathrm{s}^{-1}$. The brighter sources are con- sistent with distributions $\propto \rho_{c}^{1.5} r_{c}^{2}$ or $\propto \rho_{c}^{1.08} r_{c}^{2}\left(Z / Z_{\odot}\right)^{0.33 \pm 0.1}$, as suggested for bright LMXBs by Verbunt \& Hut (1987) and Jordán et al. (2004), while the fainter sources require a lesser dependence on density and/or metallicity. Considering the looseness of the constraints on the brighter sources, it is still possible that the two groups arise from the same distribution; studies of additional clusters will allow this to be tested. There are several possible reasons for a difference: The bright hard sources may contain a substantial number of qLMXBs and/or MSPs, with a different distribution from CVs (Wijnands et al. 2005). The faint hard sources may include large numbers of active binaries or primordial CVs with a lower density distribution (e.g., Bassa et al. 2004). Finally, the densest clusters will produce many $\mathrm{CVs}$ and destroy them relatively quickly, due to the short timescale for their next interaction (e.g., Verbunt 2003). When formed (typically from turnoff stars at $\sim 0.8 M_{\odot}$ ), these $\mathrm{CVs}$ will be relatively brighter than after a few billion years, so the densest clusters should have relatively more bright CVs. These effects can also be seen in the slope of the X-ray luminosity function (Pooley et al. 2002b), which flattens for the densest clusters.

\section{CONCLUSION}

Terzan 5 contains $28 \mathrm{X}$-ray sources above $L_{\mathrm{X}}=10^{32} \mathrm{ergs} \mathrm{s}^{-1}$ $(0.5-2.5 \mathrm{keV})$, the richest population of X-ray sources so far observed in a globular cluster in this $L_{\mathrm{X}}$ range. Twelve sources show soft X-ray colors suggesting a qLMXB nature. However, these sources are not generally well fit by a simple hydrogenatmosphere model, indicating that if these sources are qLMXBs, they have a substantial flux from harder nonthermal spectral components (as seen in noncluster systems; e.g., Rutledge et al. 2001). Several faint X-ray sources have demonstrated substantial variability, up to a factor of 5, between the 2000 and 2003 Chandra observations.

We constructed an X-ray color-color diagram for the sources in Terzan 5, for comparison with X-ray sources of similar luminosity 
at the Galactic center. We find that the X-ray colors of the Galactic center sources are substantially harder than the relatively hard $\mathrm{X}$-ray sources in Terzan 5, even when controlling for the differences in photoelectric absorption. This suggests an intrinsic difference between the sources in Terzan 5 and the Galactic center.

Our study of the distribution of X-ray sources among globular clusters finds that likely qLMXBs and hard X-ray sources with $L_{\mathrm{X}}>10^{32} \mathrm{ergs} \mathrm{s}^{-1}$ (which may be dominated by bright CVs) show consistency with the parameterizations by density, core radius, and metallicity of Verbunt \& Hut (1987) and Jordán et al. (2004). However, the hard X-ray sources with $10^{31} \mathrm{ergs} \mathrm{s}^{-1}<$
$L_{\mathrm{X}}<10^{32} \mathrm{ergs} \mathrm{s}^{-1}$ show a lesser dependence on density and metallicity.

C. O. H. is supported by the Lindheimer Postdoctoral Fellowship at Northwestern University. We are grateful to George Rybicki and Ramesh Narayan for the use of their NSATMOS code. We acknowledge very useful conversations with Scott Ransom, Mike Muno, Ron Taam, and Jae Sub Hong and the assistance of Patrick Broos and the Chandra X-Ray Center.
Bassa, C., et al. 2004, ApJ, 609, 755

Becker, W., et al. 2003, ApJ, 594, 798

Bellazzini, M., Pasquali, A., Federici, L., Ferraro, F. R., \& Pecci, F. F. 1995, ApJ, 439, 687

Bergbusch, P. A., \& Van den Berg, D. A. 2001, ApJ, 556, 322

Bogdanov, S., Grindlay, J. E., Heinke, C. O., Camilo, F., Freire, P. C. C., \& Becker, W. 2006, ApJ, 646, 1104

Bogdanov, S., Grindlay, J. E., \& van den Berg, M. 2005, ApJ, 630, 1029

Cackett, E. M., et al. 2006, MNRAS, 369, 407

Campana, S., Colpi, M., Mereghetti, S., Stella, L., \& Tavani, M. 1998, A\&A Rev., 8, 279

Clark, G. W. 1975, ApJ, 199, L143

Cohn, H. N., Lugger, P. M., Grindlay, J. E., \& Edmonds, P. D. 2002, ApJ, 571, 818

Cool, A. M., Grindlay, J. E., Cohn, H. N., Lugger, P. M., \& Slavin, S. D. 1995, ApJ, 439, 695

Cool, A. M., Haggard, D., \& Carlin, J. L. 2002, in ASP Conf. Ser. 265, Omega Centauri: A Unique Window into Astrophysics, ed. F. van Leeuwen, J. D. Hughes, \& G. Piotto (San Francisco: ASP), 277

Damiani, F., Maggio, A., Micela, G., \& Sciortino, S. 1997, ApJ, 483, 370

Edmonds, P. D., Gilliland, R. L., Heinke, C. O., \& Grindlay, J. E. 2003, ApJ, 596, 1177

Edmonds, P. D., Grindlay, J. E., Cool, A., Cohn, H., Lugger, P., \& Bailyn, C. 1999, ApJ, 516, 250

Feigelson, E. D., Broos, P., Gaffney, J. A., Garmire, G., Hillenbrand, L. A., Pravdo, S. H., Townsley, L., \& Tsuboi, Y. 2002, ApJ, 574, 258

Freeman, P. E., Kashyap, V., Rosner, R., \& Lamb, D. Q. 2002, ApJS, 138, 185

Fruchter, A. S., \& Goss, W. M. 2000, ApJ, 536, 865

Gendre, B., Barret, D., \& Webb, N. A. 2003, A\&A, 400, 521

Giacconi, R., et al. 2001, ApJ, 551, 624

Gratton, R. G., Bragaglia, A., Carretta, E., Clementini, G., Desidera, S., Grundahl, F., \& Lucatello, S. 2003, A\&A, 408, 529

Grindlay, J. E. 1987, in IAU Symp. 125, The Origin and Evolution of Nuetron Stars, ed. D. J. Helfand \& J.-H. Huang (Dordrecht: Reidel), 173

Grindlay, J. E., Camilo, F., Heinke, C. O., Edmonds, P. D., Cohn, H., \& Lugger, P. 2002, ApJ, 581, 470

Grindlay, J. E., Cool, A. M., Callanan, P. J., Bailyn, C. D., Cohn, H. N., \& Lugger, P. M. 1995, ApJ, 455, L47

Grindlay, J. E., Heinke, C., Edmonds, P. D., \& Murray, S. S. 2001a, Science, 292, 2290

Grindlay, J. E., Heinke, C. O., Edmonds, P. D., Murray, S. S., \& Cool, A. M. 2001b, ApJ, 563, L53

Grindlay, J. E., Hertz, P., Steiner, J. E., Murray, S. S., \& Lightman, A. P. 1984, ApJ, 282, L13

Hambly, N. C., et al. 2001, MNRAS, 326, 1279

Harris, W. E. 1996, AJ, 112, 1487

Heinke, C. O., Edmonds, P. D., Grindlay, J. E., Lloyd, D. A., Cohn, H. N., \& Lugger, P. M. 2003a, ApJ, 590, 809

Heinke, C. O., Grindlay, J. E., \& Edmonds, P. D. 2005a, ApJ, 622, 556

Heinke, C. O., Grindlay, J. E., Edmonds, P. D., Cohn, H. N., Lugger, P. M., Camilo, F., Bogdanov, S., \& Freire, P. C. 2005b, ApJ, 625, 796

Heinke, C. O., Grindlay, J. E., Lloyd, D. A., \& Edmonds, P. D. 2003b, ApJ, 588,452
Heinke, C. O., Grindlay, J. E., Lugger, P. M., Cohn, H. N., Edmonds, P. D., Lloyd, D. A., \& Cool, A. M. 2003c, ApJ, 598, 501

Heinke, C. O., Rybicki, G. B., Narayan, R., \& Grindlay, J. E. 2006, ApJ, 644, 1090

Hessels, J. W. T., Ransom, S. M., Stairs, I. H., Freire, P. C. C., Kaspi, V. M., \& Camilo, F. 2006, Science, 311, 1901

in 't Zand, J. J. M., van Kerkwijk, M. H., Pooley, D., Verbunt, F., Wijnands, R., \& Lewin, W. H. G. 2001, ApJ, 563, L41

Ivanova, N. 2006, ApJ, 636, 979

Ivanova, N., Fregeau, J. M., \& Rasio, F. A. 2005, in ASP Conf. Ser. 328, Binary Radio Pulsars, ed. F. A. Rasio \& I. H. Stairs (San Francisco: ASP), 231 Johnston, H. M., \& Verbunt, F. 1996, A\&A, 312, 80

Jonker, P. G., Galloway, D. K., McClintock, J. E., Buxton, M., Garcia, M., \& Murray, S. 2004, MNRAS, 354, 666

Jordán, A., et al. 2004, ApJ, 613, 279

Kong, A. K. H., Bassa, C., Pooley, D., Lewin, W. H. G., Homer, L., Verbunt, F., Anderson, S. F., \& Margon, B. 2006, ApJ, 647, 1065

Kundu, A., Maccarone, T. J., \& Zepf, S. E. 2002, ApJ, 574, L5

Kundu, A., Maccarone, T. J., Zepf, S. E., \& Puzia, T. H. 2003, ApJ, 589, L81

Liedahl, D. A., Osterheld, A. L., \& Goldstein, W. H. 1995, ApJ, 438, L115

Lugger, P. M., Cohn, H. N., Heinke, C. O., Grindlay, J. E., \& Edmonds, P. D. 2006, ApJ, in press

Maccarone, T. J., Kundu, A., \& Zepf, S. E. 2003, ApJ, 586, 814

- 2004, ApJ, 606, 430

Makishima, K., et al. 1981, ApJ, 247, L23

Monet, D. G., et al. 2003, AJ, 125, 984

Muno, M. P., et al. 2003, ApJ, 589, 225

Origlia, L., \& Rich, R. M. 2004, AJ, 127, 3422

Patterson, J., \& Raymond, J. C. 1985, ApJ, 292, 535

Pooley, D., \& Hut, P. 2006, ApJ, 646, L143

Pooley, D., et al. 2003, ApJ, 591, L131

. 2002a, ApJ, 569, 405

2002b, ApJ, 573, 184

Predehl, P., Costantini, E., Hasinger, G., \& Tanaka, Y. 2003, Astron. Nachr., 324, 73

Press, W. H., Teukolsky, S. A., Vetterling, W. T., \& Flannery, B. P. 1992, Numerical Recipes in FORTRAN (2nd ed.; Cambridge: Cambridge Univ. Press)

Ransom, S. M., Hessels, J. W. T., Stairs, I. H., Freire, P. C. C., Camilo, F., Kaspi, V. M., \& Kaplan, D. L. 2005, Science, 307, 892

Rutledge, R. E., Bildsten, L., Brown, E. F., Pavlov, G. G., \& Zavlin, V. E. 2001, ApJ, 551, 921

. 2002, ApJ, 578, 405

Sarazin, C. L., Kundu, A., Irwin, J. A., Sivakoff, G. R., Blanton, E. L., \& Randall, S. W. 2003, ApJ, 595, 743

Verbunt, F. 2003, in ASP Conf. Ser. 296, New Horizons in Globular Cluster Astronomy, ed. G. Piotto et al. (San Francisco: ASP), 245

Verbunt, F., \& Hut, P. 1987, in IAU Symp. 125, The Origin and Evolution of Neutron Stars, ed. D. J. Helfand \& J.-H. Huang (Dordrecht: Reidel), 187

Verbunt, F., \& Lewin, W. H. G. 2006, in Compact Stellar X-Ray Sources, ed. W. H. G. Lewin \& M. van der Klis (Cambridge: Cambridge Univ. Press), 341 Wijnands, R., Heinke, C. O., Pooley, D., Edmonds, P. D., Lewin, W. H. G., Grindlay, J. E., Jonker, P. G., \& Miller, J. M. 2005, ApJ, 618, 883 